\title{
SLIT3 deficiency attenuates pressure overload-induced cardiac fibrosis and remodeling
}

Lianghui Gong, ${ }^{1,2}$ Shuyun Wang, ${ }^{1}$ Li Shen, ${ }^{1}$ Catherine Liu, ${ }^{1}$ Mena Shenouda, ${ }^{1}$ Baolei Li, ${ }^{1}$ Xiaoxiao Liu, John A. Shaw, ${ }^{3}$ Alan L. Wineman, ${ }^{4}$ Yifeng Yang, ${ }^{2}$ Dingding Xiong, ${ }^{1}$ Anne Eichmann, $, 5,6,7$

Sylvia M. Evans, ${ }^{8,9,10}$ Stephen J. Weiss, ${ }^{11,12,13,14,15}$ and Ming-Sing $\mathrm{Si}^{1}$

'Section of Pediatric Cardiovascular Surgery, Department of Cardiac Surgery, Michigan Medicine, University of Michigan, Ann Arbor, Michigan, USA. ${ }^{2}$ Department of Cardiovascular Surgery, The Second Xiangya Hospital of Central South University, Changsha, China. ${ }^{3}$ Department of Aerospace Engineering and ${ }^{4}$ Department of Mechanical Engineering, University of Michigan, Ann Arbor, Michigan, USA. ${ }^{5}$ Yale Cardiovascular Research Center, Department of Internal Medicine, Yale University School of Medicine, New Haven, Connecticut, USA. ${ }^{6}$ Paris Cardiovascular Research Center, INSERM U970, Paris, France. 'Department of Cellular and Molecular Physiology, Yale University School of Medicine, New Haven, Connecticut, USA. ${ }^{8}$ Skaggs School of Pharmacy and Pharmaceutical Sciences, ${ }^{9}$ Department of Medicine, and ${ }^{10}$ Department of Pharmacology, UCSD, La Jolla, California, USA. "Division of Genetic Medicine, ${ }^{12}$ Department of Internal Medicine, ${ }^{13}$ Life Sciences Institute, ${ }^{14}$ Cellular and Molecular Biology Graduate Program, and ${ }^{15}$ Rogel Cancer Center, Michigan Medicine, University of Michigan, Ann Arbor, Michigan, USA.

In pulmonary hypertension and certain forms of congenital heart disease, ventricular pressure overload manifests at birth and is an obligate hemodynamic abnormality that stimulates myocardial fibrosis, which leads to ventricular dysfunction and poor clinical outcomes. Thus, an attractive strategy is to attenuate the myocardial fibrosis to help preserve ventricular function. Here, by analyzing RNA-sequencing databases and comparing the transcript and protein levels of fibrillar collagen in WT and global-knockout mice, we found that slit guidance ligand 3 (SLIT3) was present predominantly in fibrillar collagen-producing cells and that SLIT3 deficiency attenuated collagen production in the heart and other nonneuronal tissues. We then performed transverse aortic constriction or pulmonary artery banding to induce left and right ventricular pressure overload, respectively, in WT and knockout mice. We discovered that SLIT3 deficiency abrogated fibrotic and hypertrophic changes and promoted long-term ventricular function and overall survival in both left and right ventricular pressure overload. Furthermore, we found that SLIT3 stimulated fibroblast activity and fibrillar collagen production, which coincided with the transcription and nuclear localization of the mechanotransducer yes-associated protein 1 . These results indicate that SLIT3 is important for regulating fibroblast activity and fibrillar collagen synthesis in an autocrine manner, making it a potential therapeutic target for fibrotic diseases, especially myocardial fibrosis and adverse remodeling induced by persistent afterload elevation.

Conflict of interest: The authors have declared that no conflict of interest exists.

Copyright: ( 2020 , American Society for Clinical Investigation.

Submitted: February 13, 2020

Accepted: May 6, 2020

Published: June 18, 2020.

Reference information: JCI Insight. 2020;5(12):e136852.

https://doi.org/10.1172/jici.

insight.136852.

\section{Introduction}

Neonatal pulmonary hypertension and certain forms of complex congenital heart disease subject the cardiac ventricle to pressure overload early in life (1-3). Although this hemodynamic stress is well tolerated in the short term, chronic elevation in afterload can result in adverse ventricular remodeling characterized by fibrosis and hypertrophy, which then contributes to ventricular dysfunction $(4,5)$. This is especially important in systemic right ventricle congenital heart disease and pediatric primary pulmonary hypertension, where patient survival is determined by ventricular function $(1,6,7)$. Therefore, in these clinical conditions where chronic pressure overload is obligate and unavoidable, preventing the adverse remodeling response would have an important clinical benefit (8).

Mammalian slit guidance ligands (Slit1-3) are highly conserved, secreted glycoproteins that were originally described to mediate repulsive axonal guidance during central nervous system development by binding to Roundabout (ROBO) receptors (9-12). Interestingly, SLIT3 is primarily expressed in nonneuronal 
tissues (13-16). The most striking manifestation of SLIT3 deficiency in mice is central congenital diaphragmatic hernia $(\mathrm{CDH})(13,17)$ and osteopenia $(14,18)$, while in humans, genetic variants and low SLIT3 serum levels have been associated with height (19) and osteoporosis (18), respectively.

Fibrillar collagen is the major component of extracellular matrix (ECM) in a variety of load-bearing tissues, including the central diaphragm tendon, bone, and heart $(20,21)$. In the developing heart, SLIT3 is intensively expressed in mesenchymal cushions, and ultimately transforms into dense connective tissue, including the membranous ventricular septum as well as the atrioventricular and semilunar valves (22). Correspondingly, membranous ventricular septum defects, as well as atrioventricular and aortic valve abnormalities, are exhibited in SLIT3-mutant mice $(22,23)$. Congenital heart disease involving tetralogy of Fallot and septal and outflow tract defects is associated with SLIT3 variants in humans (24).

Considering the abovementioned association between SLIT3 deficiency and these collagen-rich tissue defects, we sought to further understand the function of SLIT3 in postnatal nonneuronal tissues, especially in the heart, where excessive fibrillar collagen accumulation leads to myocardial fibrosis and maladaptive remodeling. We found that SLIT3 is present at high levels in fibrillar collagen-producing cells, and SLIT3 deficiency reduces Colla1 and Col3a1 transcript levels as well as the total collagen content in multiple nonneuronal tissues. More importantly, Slit3-targeted mice are markedly protected from pressure overloadinduced left and right ventricular fibrosis and remodeling as well as associated mortality. These results identify the axon guidance molecule SLIT3 as a fibroblast-secreted, collagen-regulating factor to promote type I collagen expression and as a potential therapeutic target for attenuating fibrillar collagen accumulation and adverse cardiac remodeling induced by pressure overload.

\section{Results}

SLIT3 is present at high levels in fibrillar collagen-producing cells. Fibrillar collagen is a subgroup of the collagen family, participates in the formation of striated fibrils, and provides robust mechanical strength (25). Collagen types I and III are the most common fibrillar collagens and are the major components of the myocardial collagen matrix (26). As the biosynthesis of fibrillar collagen is highly regulated at the transcript level (27), and SLIT3 deficiency affects collagen-rich tissues $(13,14,17,18)$, we first interrogated the cellular transcript levels of SLITs, ROBOs, COL1A1, and COL3A1 in the FANTOM5 (human) (28) and Tabula Muris (Mus musculus) (29) projects. We found that SLIT3 transcripts were present at high levels in human fibroblasts, synoviocytes, tenocytes, adipocytes, mesenchymal cells, and smooth muscle cells (Supplemental Table 1; supplemental material available online with this article; https://doi.org/10.1172/ jci.insight.136852DS1), all of which belong to the family of connective tissue cells distinguished by their ability to synthesize and deposit type I/III collagen (30). Linear regression analysis between the transcript levels of SLIT3 and COL1A1 exhibited a positive correlation across 512 human cell lines $\left(R^{2}=0.1764, P<\right.$ $10^{-15}$ ) (Figure 1A and Supplemental Figure 1A). Also, in the single-cell RNA-Seq study of mouse tissues, Slit3 transcripts were present at high levels in mesenchymal stem cells, stromal cells, and fibroblasts, and Slit3, Colla1, and Col3a1 appeared to be coexpressed in the same cell populations (Figure 1B and Supplemental Figure 1, B and C).

To support the above sequencing results by real-time PCR, we isolated and cultured primary aortic adventitial, cardiac, and pulmonary fibroblasts, as well as aortic vascular smooth muscle cells from adult WT CD-1 mice. Similar to the expression pattern of Col1a1, Slit3 transcript levels were low in left ventricular (LV) tissue and negligible in freshly isolated cardiomyocytes, fibroblasts from the heart, and other tissues yet were significantly higher in aortic vascular smooth muscle cells (Figure 1C). Interestingly, the expression pattern of Robo1, which is considered the receptor for SLIT3-mediated effects on fibrillar collagen formation in bone $(14,18)$, is different from that of Slit3 by sequencing or real-time PCR. Compared with the highly concentrated and overlapping expressions of Slit 3 and Col1a1, the expression of Robo1 expands beyond these cell populations (Figure 1, A-C; and Supplemental Figure 1, A-C). In addition, we supported SLIT3 and ROBO1 protein expression in adult primary murine fibroblasts by immunocytochemistry (Figure 1D and Supplemental Figure 8). These results indicate that fibrillar collagen-producing fibroblasts express SLIT3 and possess its primary receptor, ROBO1 (14, 17, 18, 22, 23, 31), indicating a possible autocrine axis.

Phenotypic manifestations of Slit3 $3^{-/}$CD1 mice. Due to the widespread distribution of fibrillar collagenproducing cells and the critical importance of fibrillar collagen in providing structural support for high-pressure structures, such as the cardiac ventricles, we sought to investigate the influence of SLIT3 deficiency on the postnatal cardiovascular system. Since previous studies in inbred Slit3 ${ }^{-/-}$(knockout) mice precluded 
A
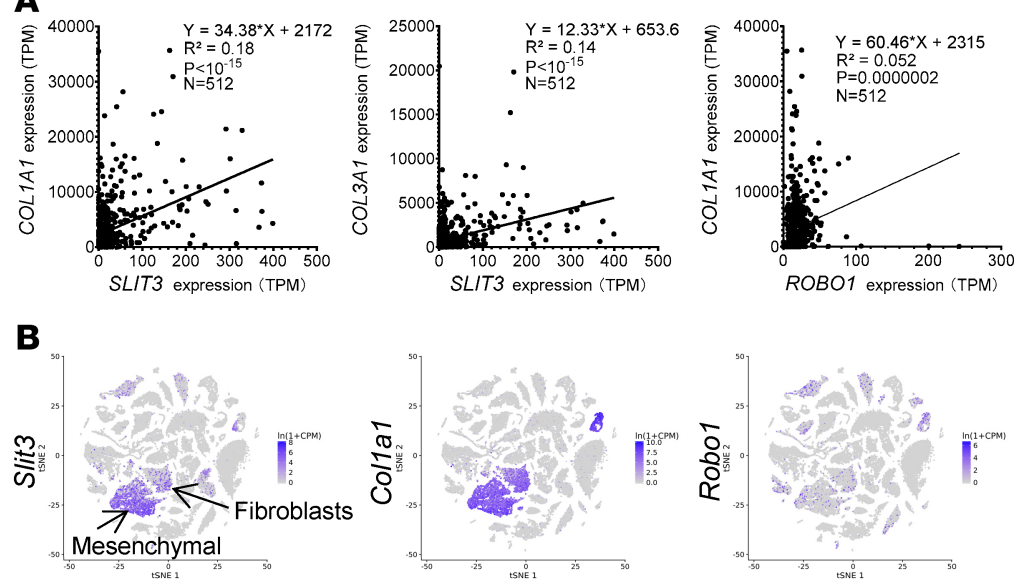

C
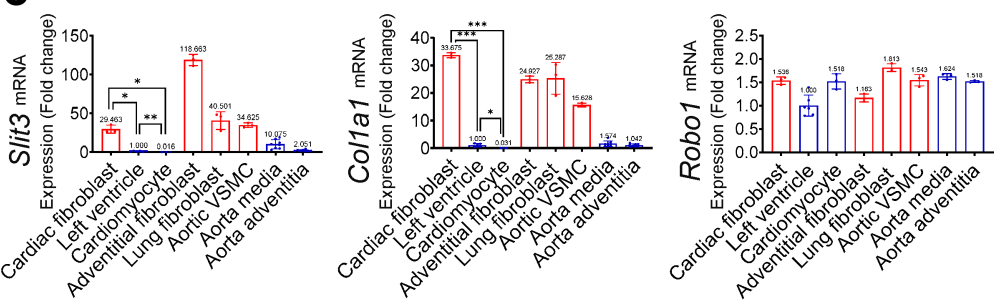

D No Primary Antibody
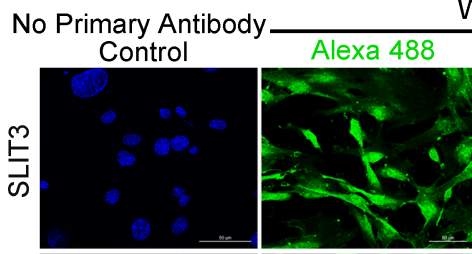

With Primary Antibody

DAPI

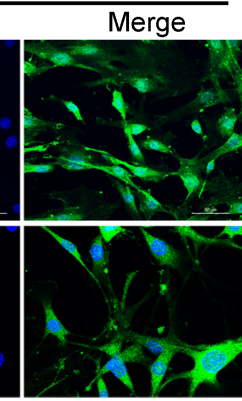

Figure 1. SLIT3 is present at high levels in fibrillar collagen-producing cells. (A) Linear regression analysis between the transcript levels of SLIT3 and COL1A1, SLIT3 and COL3A1, and ROBO1 and COL1A1 across 512 cell lines from the FANTOM5 project (28). TPM, transcripts per million. (B) Single-cell transcriptome data from the Tabula Muris project (29), with t-distributed stochastic neighbor embedding plot of all cells collected by FACS, overlaid with the predominant cell type composing each cluster ( $n=44,949$ individual cells from 20 mouse organs). The clusters of cells expressing Slit3, Col1a1, and Robo1. (C) Transcript levels of Slit3, Col1a1, and Robo1 in cardiac fibroblasts, left ventricle, freshly isolated cardiomyocytes, aortic adventitial fibroblasts, lung fibroblasts, aortic vascular smooth muscle cells (VSMC), aorta media, and aorta adventitia from WT mice. Samples taken directly from or isolated from living tissue are marked blue and samples of purified and cultured cells are marked red on panels. Each data point represents tissue/cells obtained from a single animal. (D) Confocal immunofluorescence images of mouse aortic adventitial fibroblasts stained with SLIT3 (shown in green), ROBO1 (shown in green), and DAPI (shown in blue) $(n=2)$. Scale bars: $50 \mu \mathrm{m} .{ }^{*} P<0.05,{ }^{* *} P<0.01$, and ${ }^{* *} P<0.001$ using 1 -way ANOVA with Tamhane T2 multiple comparisons test (C).

analyses of the postnatal cardiovascular system because of the confounding phenotype of diaphragmatic hernia $(13,14,17,18)$, we used knockout mice on a $\mathrm{CD}-1$ (outbred) background ( $\mathrm{F} / \mathrm{M}=1: 1)$, which have a lower penetrance of central CDH (approximately 5\%, Supplemental Figure 2F) and normal LV systolic function in the first year of life (Supplemental Table 2). Compared with age-matched WT mice, the body weight, tibia length, and hair follicle density of Slit $3^{-/-}$mice were all significantly reduced (Supplemental Figure 2, A-E). Further, the amounts of fibrillar collagen fibers found in the arterial adventitia and perivascular/interstitial regions of multiple nonneuronal organs (Figure 2A), including those of the heart (Figure 3, A and E), were reduced in Slit3 ${ }^{-/-}$mice while myocardial capillary density was preserved (Figure 3A). Interestingly, previous studies in inbred Slit $3^{-1-}$ mice described that the liver was always found in the hernia 
A
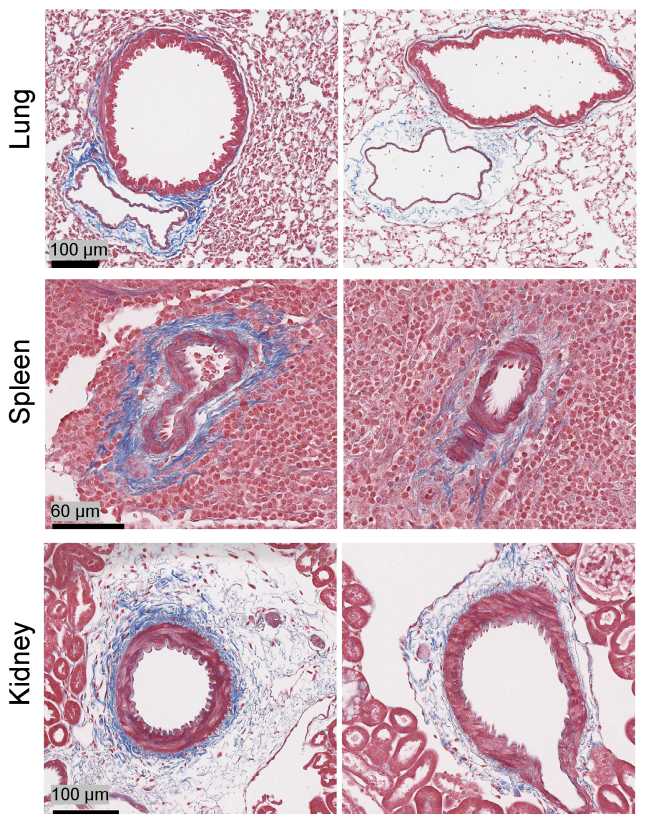

B
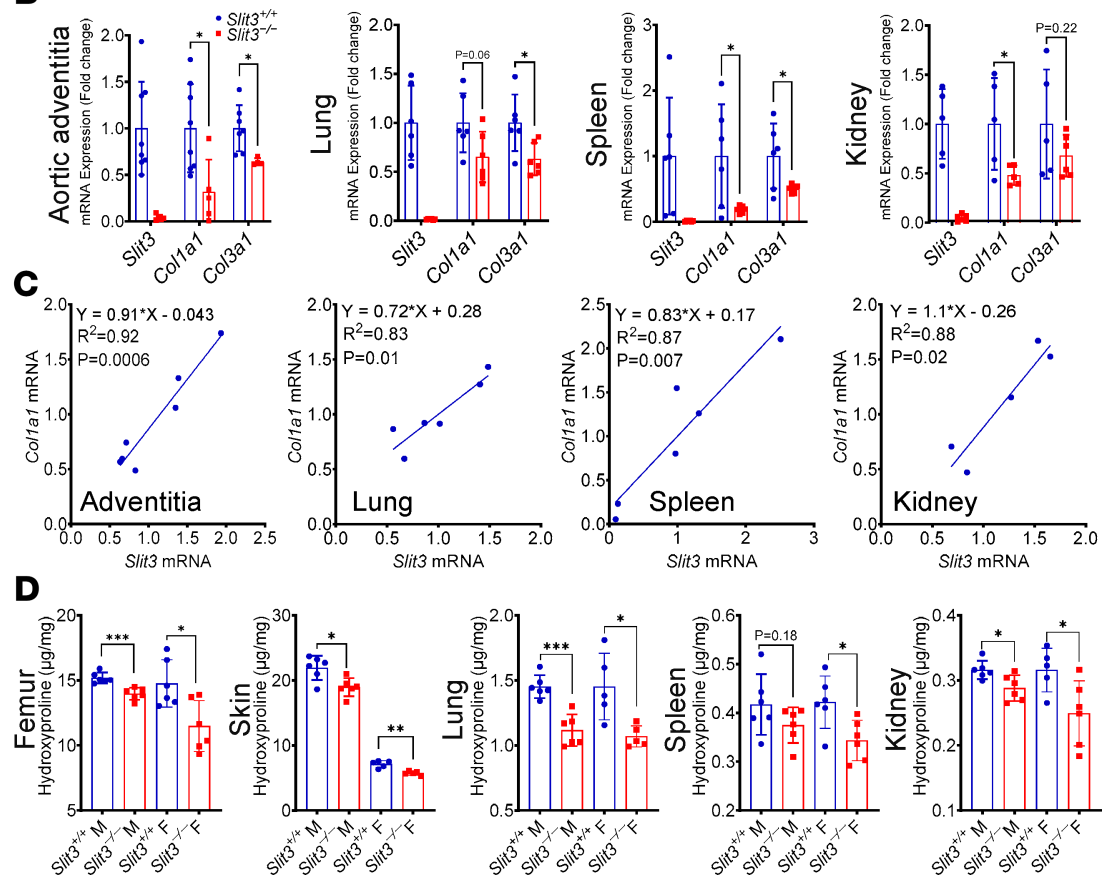

Figure 2. SLIT3 deficiency reduces fibrillar collagen production in vivo. (A) Masson's trichrome-stained histological sections of lung, spleen, and kidney in 8-week-old Slit3 ${ }^{-\%}$ and WT mice. Results are representative of samples obtained from 5 animals per genotype group. Scale bars: $100 \mu \mathrm{m}, 60 \mu \mathrm{m}$, and $100 \mu \mathrm{m}$ from top to bottom. (B) Transcript levels of Slit3, Col1a1, and Col3a1 in the aortic adventitia, lung, spleen, and kidney in 8-week-old Slit3 ${ }^{-\%}$ and WT mice ( $n=4-6$ per group). (C) Linear regression analysis between the transcript levels of Slit3 and Col1a1 in the aortic adventitia, lung, spleen, and kidney from 8-week-old WT mice ( $n=5-7$ per group). (D) Quantification of tissue collagen content by assessment of hydroxyproline concentrations in the femur, skin, lung, spleen, and kidney in 8-week-old Slit3 ${ }^{-1-}$ and WT mice $\left(n=5-8\right.$ per group). Data are presented as mean \pm SD. ${ }^{*} P<0.05,{ }^{* *} P<0.01$, and ${ }^{* * *} P<$ 0.001 vs. WT mice using the unpaired 2-tailed Student's $t$ test (B and D). $n$, number; M, male; F, female.

sac and adhered to its wall without the formation of the falciform ligament $(13,17)$. However, we observed in a CD-1-knockout mouse that the stomach, rather than the liver, was the only hernia sac content (Supplemental Figure 2, F-H), which suggests that the formation of central CHD is not necessarily related to liver or falciform ligament development.

SLIT3 deficiency reduces fibrillar collagen production in vivo and affects LV biomechanical toughness. Given the histological changes observed in Slit $3^{-/-}$mice, we next evaluated Colla1 and Col3a1 transcript levels as well as total collagen protein levels in the aortic adventitia, lung, spleen, kidney, bone, skin, and heart. Collal and Col3a1 transcripts (Figure 2B and Figure 3B), as well as hydroxyproline levels (Figure 2D and Figure $3 \mathrm{~F}$ ), were all significantly reduced in Slit $3^{-/-}$compared with WT mice. The transcript levels of Slit3 and Colla1 among individual WT mice exhibited a strong positive and linear correlation in all analyzed tissues (Figure 2C and Figure 3C). Interestingly, the indexed femur and heart weight (normalized to tibia length), as well as cardiomyocyte size, were also decreased in Slit3 ${ }^{-/-}$mice (Figure 3I and Supplemental Figure 6A).

Because cardiac fibroblasts are responsible for most of the fibrillar collagen production in the heart and are widely distributed in the left and right ventricles, which have different embryological origins and physiological characteristics $(32,33)$, we next determined whether there was a ventricle-specific expression pattern for Slit3. Although the ventricles possessed widely varying levels of Slit3 transcripts among animals, we found that Slit3 transcript levels were strongly correlated in the right ventricle and left ventricle in the same individual $\left(R^{2}=0.98\right.$, Figure 3D), indicating a tightly regulated balance of Slit 3 ventricular expression, which is also consistent with the common embryological origin of fibroblasts in both ventricles (34-36). Because SLIT3 deficiency reduces the myocardial fibrillar collagen content, which plays crucial mechanical roles in the ventricle, we then investigated its effect on LV toughness, i.e., the amount of energy absorbed before irreversibly fracturing/tearing. Using gradual inflation of the cardioplegia-relaxed LV, the energy density required to rupture the left ventricles from Slit $3^{--}$mice was significantly reduced compared with WT mice (Figure 3G). Consistently, in 1-year-old mice, SLIT3 deficiency increased mitral E/A ratio, which is a sensitive indicator of LV compliance (Figure 3, E-H, and ref. 37). To further support these 
A Slit3 $^{++}$
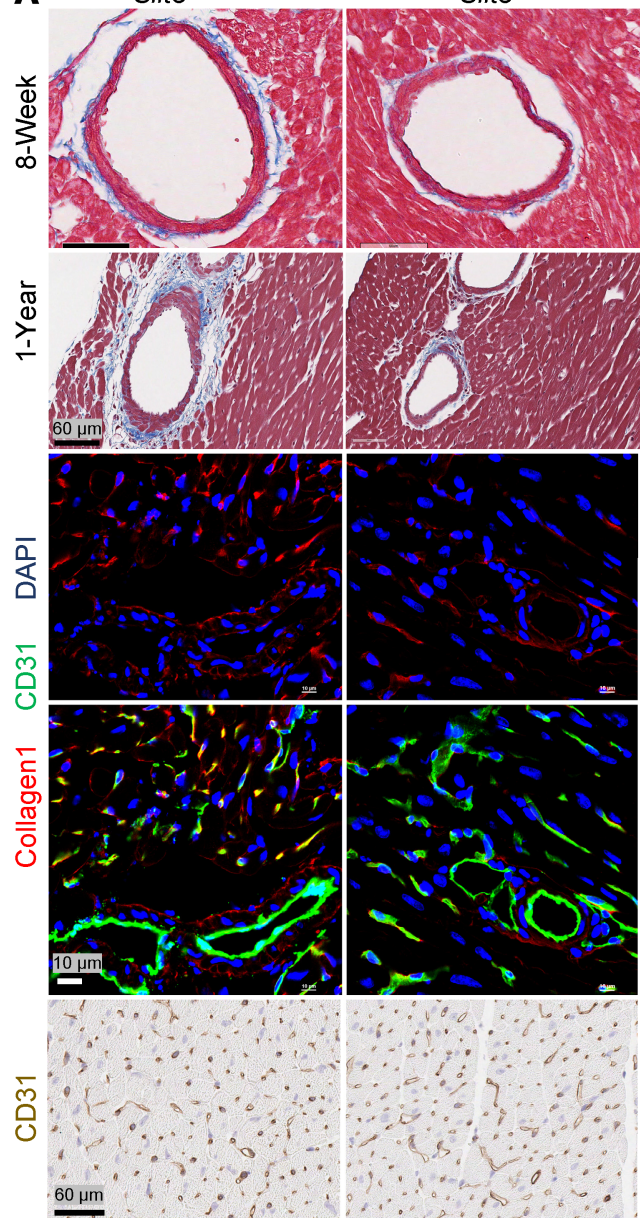

Slit3-1-
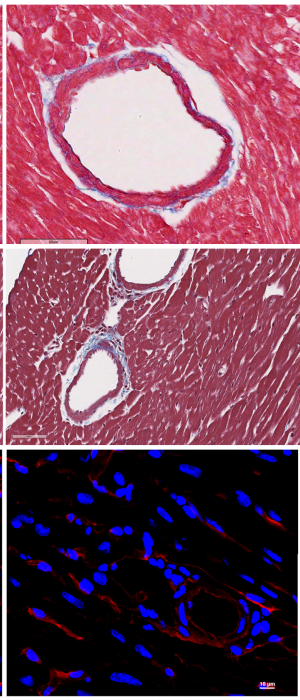

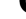

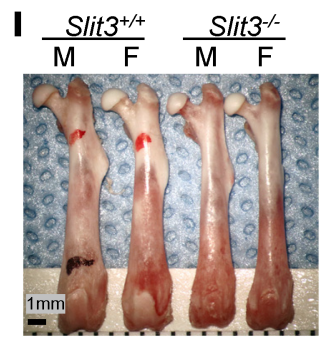

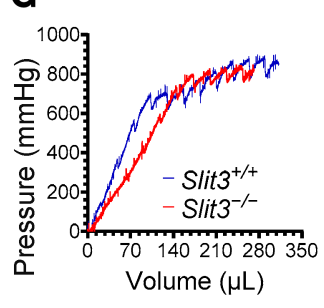

D

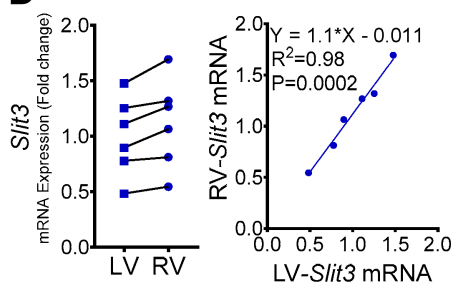

G

B
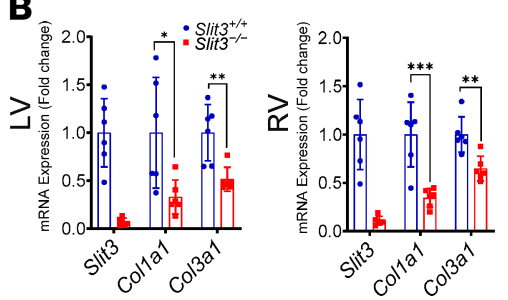

E
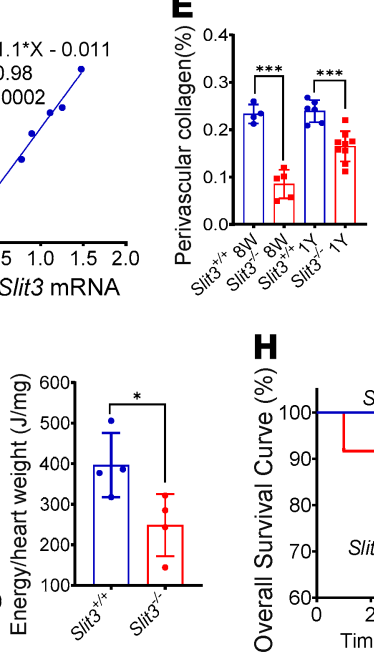

C
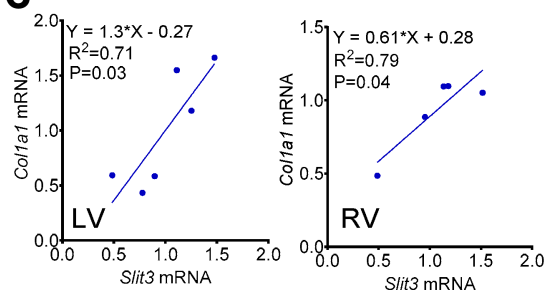

Slit3 mRNA

F
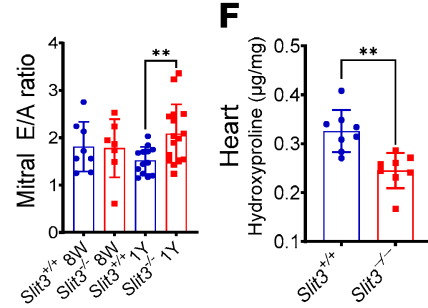
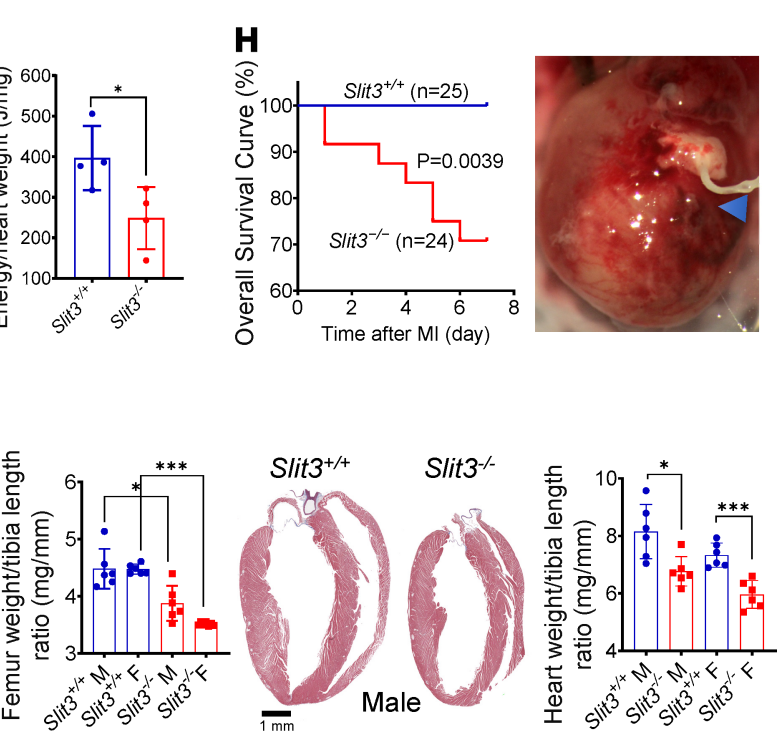

Figure 3. SLIT3 deficiency reduces cardiac fibrillar collagen production and LV biomechanical toughness. (A) Representative Masson's trichrome-stained histological sections of heart in 8-week-old and 1-year-old Slit $3^{\%}$ and WT mice; representative confocal immunofluorescence images of heart stained with collagen type I (red), CD31 (green), and DAPI (blue) in 8-week-old Slit3\% and WT mice; and representative immunohistochemical stain for CD31 in sections of hearts from 8-week-old Slit3 ${ }^{--}$and WT mice ( $n=3$ per group). (B) Transcript levels of Slit3, Col1a1, and Col3a1 in the left ventricle and right ventricle in 8-week-old Slit3 ${ }^{-1-}$ and WT mice ( $n=4-6$ per group). (C) Linear regression analysis between the transcript levels of Slit3 and Colla1 in the left ventricle and right ventricle from 8-week-old WT mice ( $n=6$ per group). (D) Transcript levels of Slit3 in the left and right ventricle, and their linear regression analysis in 8-week-old WT mice ( $n=6$ per group). (E) Quantification of perivascular collagen area of coronary arteries and mitral ratio of peak early to late diastolic filling velocity (mitral E/A ratio) in 8-week-old and 1-year-old Slit3 ${ }^{-1-}$ and WT mice ( $n=4-16$ per group). (F) Quantification of cardiac collagen content by assessment of hydroxyproline concentrations in 8-week-old Slit3 $\%$ and WT mice (female, $n=8$ per group). (C) Representative passive LV pressure-volume curve and quantification of energy density required to rupture the cardioplegia-relaxed left ventricles by balloon catheter inflation in 8 -week-old Slit3 ${ }^{-/}$and WT mice $(n=$ 4 per group). (H) Representative image of myocardial rupture and overall survival curve of the first week after left anterior descending coronary artery ligation (myocardial infarction, MI) in 8-week-old Slit ${ }^{-\%}$ and WT mice. The blue arrowhead indicates the position of the myocardial rupture hole, and survival analysis was performed using the Kaplan-Meier method. Log-rank test, $P=0.0039$ ( $n=24-25$ per group). (I) Representative image of femurs or hearts, as well as quantification of femur or heart weight/tibia length ratio in 8 -week-old Slit3 ${ }^{--}$and WT mice $\left(n=6\right.$ per group). Scale bar: 1 mm. Data are presented as mean \pm SD. ${ }^{*} P$ $<0.05$, ${ }^{* *} P<0.01$, and ${ }^{* * *} P<0.001$ vs. WT mice using the unpaired 2-tailed Student's $t$ test (B, E-G, and I). $n$, number; M, male; F, female.

findings, we evaluated WT and knockout mice in a left anterior descending coronary artery ligation myocardial infarction (MI) model since myocardial collagen is needed to maintain the integrity of the infarcted myocardium, thereby preventing ventricular rupture $(38,39)$. We found that the 1-week survival rate was significantly reduced in Slit $3^{-/-}$compared with WT mice. Among $25 \mathrm{WT}$ and 24 Slit $3^{-/-}$mice after surgery, a total of 7 Slit $3^{-/-}$mice died in the first week, 6 of which suffered LV rupture, characterized by a large amount of blood in the thoracic cavity around the heart and an area of transmural rupture in the ischemic area of the left ventricle (Figure $3 \mathrm{H}$ ). 
A

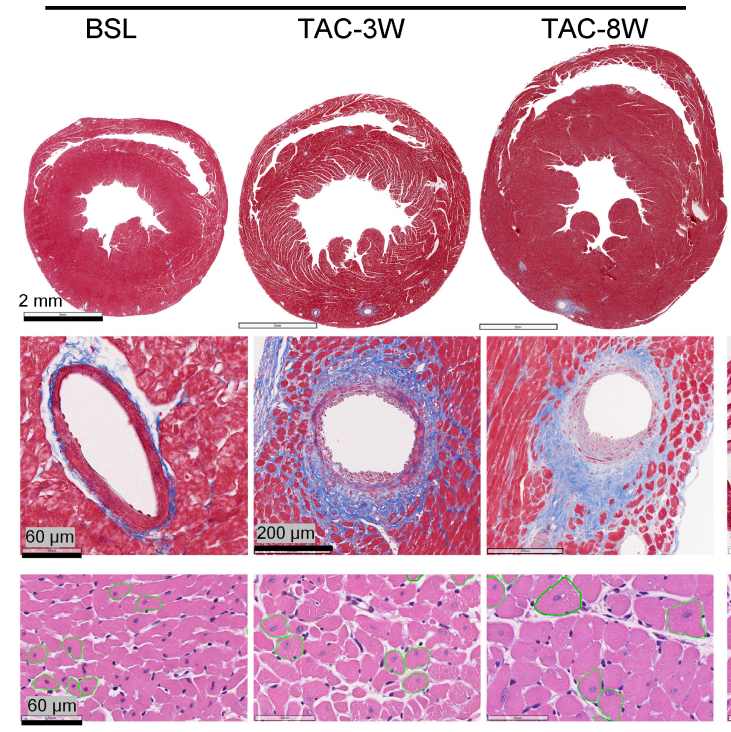

Slit3--

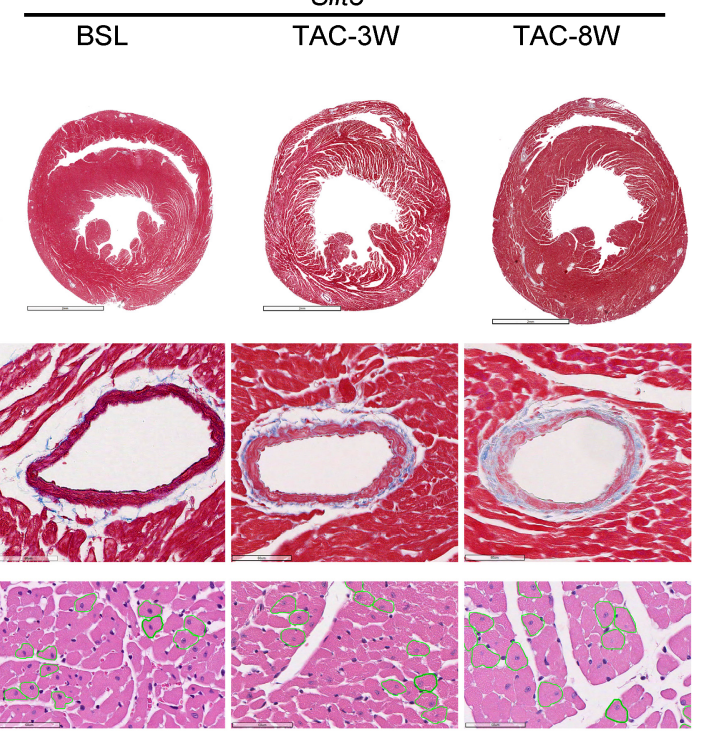

B

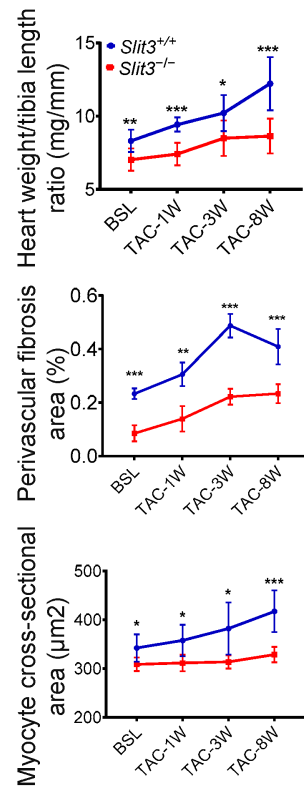

C

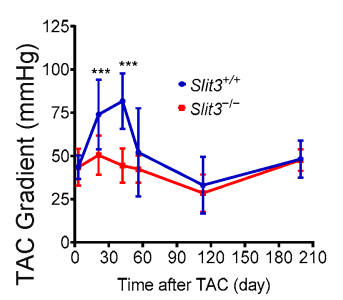

D

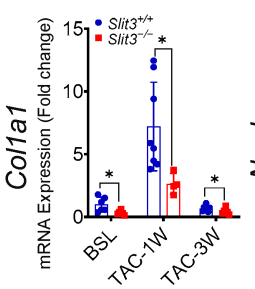

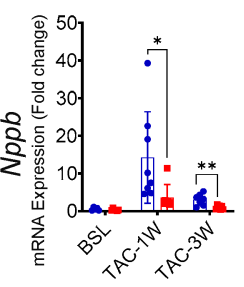

E

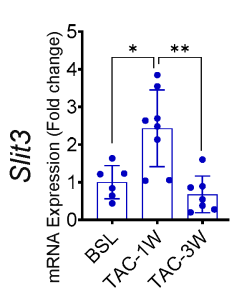

$\mathbf{F}$

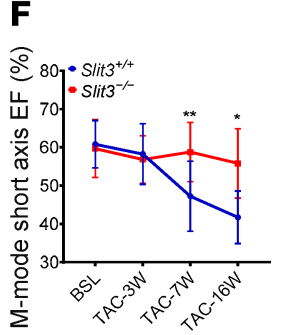

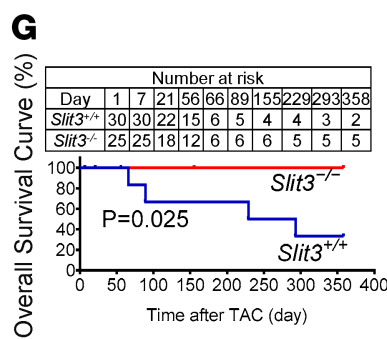

Figure 4. SLIT3 deficiency attenuates LV fibrosis and adverse remodeling. (A) Histological transverse sections of whole heart (first row, Masson's trichrome stain), coronary arteries (second row, Masson's trichrome stain), and LV myocytes (third row, hematoxylin and eosin stain) in Slit3 ${ }^{-1-}$ and WT mice before and at 3 and 8 weeks after transverse aortic constriction (TAC) $(n=6)$. Scale bars: $2 \mathrm{~mm}, 60$ or $200 \mu \mathrm{m}$, and $60 \mu \mathrm{m}$ from top to bottom. (B) Quantification of heart weight/tibia length ratio, coronary perivascular fibrosis area, and cardiomyocyte cross-sectional area in Slit3 $3^{-1}$ and WT mice before and at 1, 3, and 8 weeks after TAC ( $n=4-9$ per group). (C) TAC peak pressure gradient determined by echocardiography in Slit3 $3^{-1}$ and WT mice after surgery ( $n=4-24$ per group, initial gradients at day 3, $43 \pm 6.8 \mathrm{vs.} 43 \pm 11 \mathrm{mmHg}, P>0.99$ ). (D and E) Transcript levels of Col1a1, Nppb, and Slit3 in the left ventricle in Slit3 $3^{-/}$and WT mice before and at 1 and 3 weeks after TAC ( $n=6-8$ per group). (F) LV ejection fraction (EF) determined by echocardiography in Slit3 $3^{-1-}$ and WT mice before and at 3, 7, and 16 weeks after TAC ( $n=5-19$ per group). (C) Long-term overall survival curve of Slit $3^{-1 /}$ and WT mice from day 1 after TAC. Survival analysis was performed using the Kaplan-Meier method. Log-rank test, $P=0.0245(n=25-30$ per group). Data are presented as mean \pm SD. ${ }^{*} P<0.05$, ${ }^{* *} P<0.01$, and ${ }^{* *} P<0.001$ vs. WT mice using the unpaired 2 -tailed Student's $t$ test (B-D and $\mathbf{F}$ ) and 1-way ANOVA with Tamhane T2 multiple comparisons test (E). BSL, 7- to 9-week-old baseline mice before surgery.

SLIT3 deficiency attenuates LV fibrosis and adverse remodeling. Chronic pressure overload of the left and right ventricles occurs in acquired and congenital cardiovascular disease and leads to adverse myocardial remodeling characterized by fibrillar collagen accumulation and myocyte hypertrophy. To investigate the role of SLIT3 in the LV and right ventricular (RV) fibrotic response to pressure overload, we subjected WT and Slit $3^{-/}$body weight-matched mice to transverse aortic constriction (TAC) or pulmonary artery banding $(\mathrm{PAB}$ ) with similar initial peak pressure gradients (Figure 4C; Figure 5E; Supplemental Figure 3, A and C; Supplemental Figure 4A; and Supplemental Figure 5A).

Successful TAC in mice induced LV fibrillar collagen accumulation and cardiomyocyte hypertrophy, but this pressure overload-induced cardiac remodeling was attenuated in Slit $3^{-1-}$ mice as compared with WT mice. Perivascular fibrillar collagen area was decreased in Slit $3^{-/-}$mice at 1, 3, and 8 weeks after TAC (Figure 4, A and B), which corresponded to decreased transcript levels of fibrosis-related genes (Colla1, Col3a1, Ctgf, Fn1, and Acta2) at 1 and 3 weeks after TAC in these animals (Figure 4D and Supplemental Figure 4D). Unexpectedly, we also observed that myocardial hypertrophy was blunted in Slit3 ${ }^{-1-}$ mice after TAC (Figure 4A and Supplemental Figure 4C), consistent with a reduced heart weight/tibia length ratio; LV cardiomyocyte cross-sectional area at 1, 3, and 8 weeks after TAC (Figure 4, A and B); and LV 
A

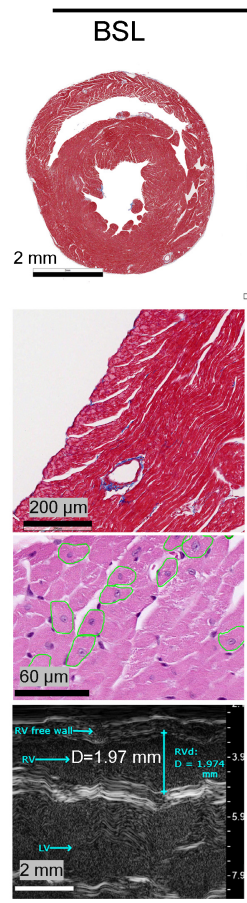

D

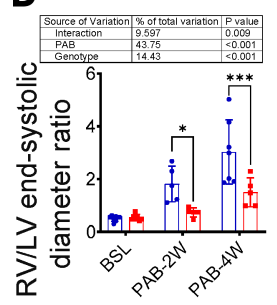

Slit3 $3^{+/+}$

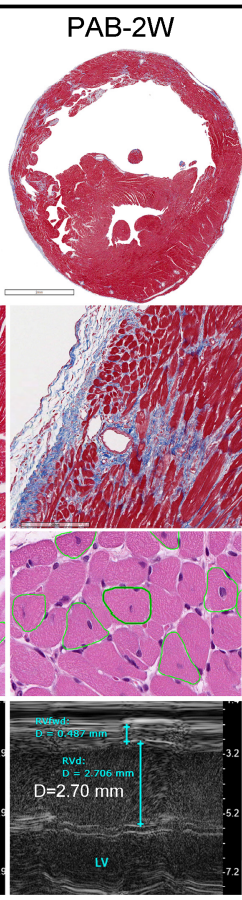

E

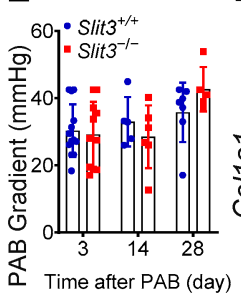

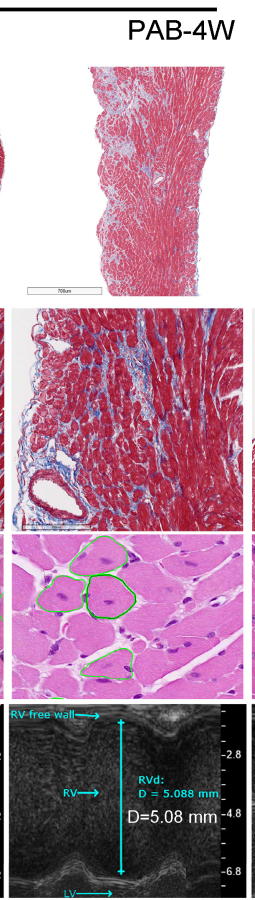

$\mathbf{F}$

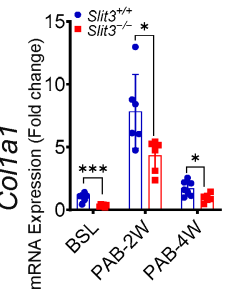

Slit3- -

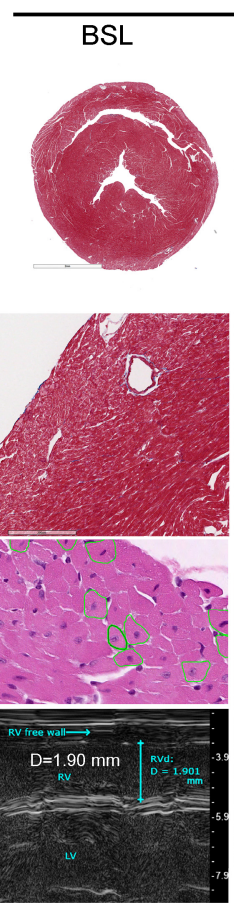

1
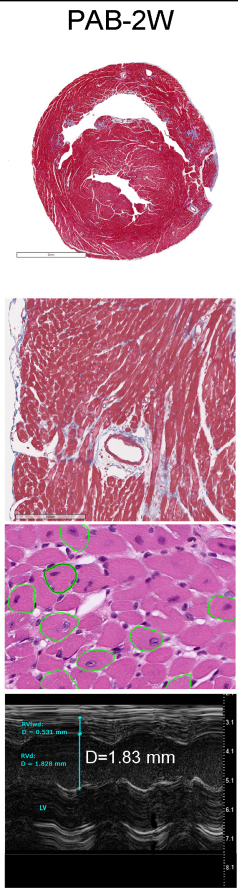

G

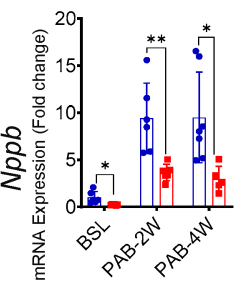

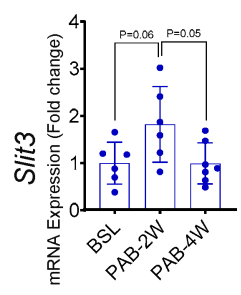

PAB-4W
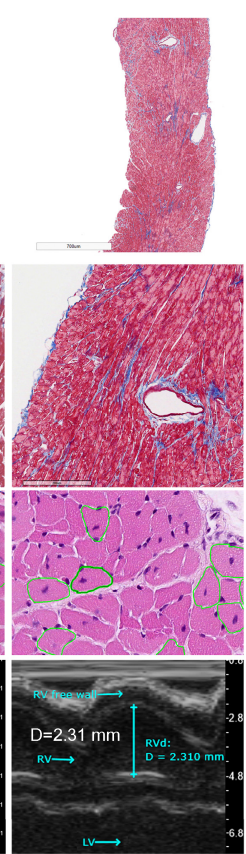

H

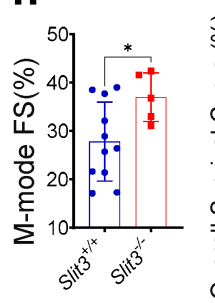

B
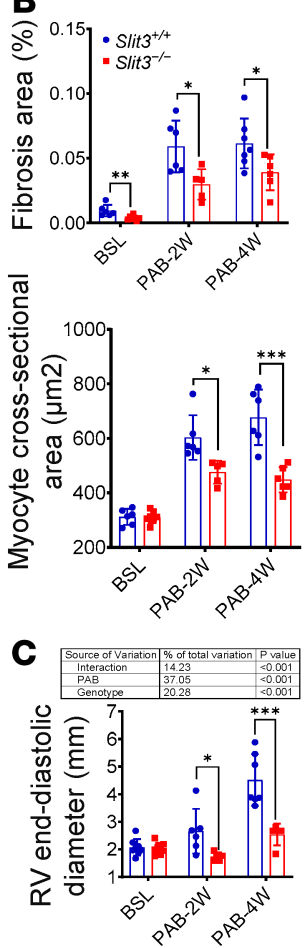

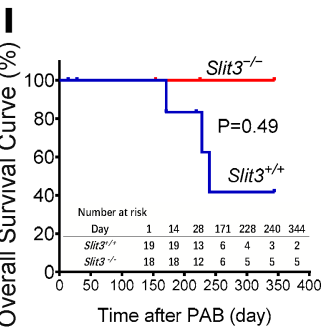

Figure 5. SLIT3 deficiency attenuates RV fibrosis and adverse remodeling. (A) Histological transverse sections of whole heart or part of RV free wall (first row, Masson's trichrome stain), and RV free wall with coronary arteries (second row, Masson's trichrome stain), RV free wall myocytes (third row, hematoxylin and eosin stain) and representative images of RV echocardiogram (fourth row, M-mode parasternal short axis) in Slit ${ }^{-/}$and WT mice before and at 2 and 4 weeks after pulmonary artery banding (PAB) $(n=6)$. Scale bars: $2 \mathrm{~mm}, 200 \mu \mathrm{m}, 60 \mu \mathrm{m}$, and $2 \mathrm{~mm}$ from top to bottom. (B-D) Quantification of RV free wall fibrosis area, myocyte cross-sectional area, as well as end-diastolic diameter area and ratio determined by echocardiogram in Slit3 ${ }^{-1-}$ and WT mice before and at 2 and 4 weeks after PAB ( $n=4-9$ per group). (E) Quantification of PAB peak pressure gradient by echocardiography in Slit3 ${ }^{-/}$and WT mice at 3,14 , and 28 days after PAB ( $n=5-13$ per group; initial gradients at day $3,30 \pm 7.9$ vs. $29 \pm 9.7 \mathrm{mmHg} ; P>0.75$ ). (F and G) Transcript levels of Col1a1, Nppb, and Slit3 in the right ventricle in Slit3 ${ }^{-1}$ and WT mice before and at 2 and 4 weeks after PAB ( $n=5-6$ per group). (H) RV fractional shortening (FS) determined by echocardiography in Slit3 ${ }^{--}$and WT mice at 2 weeks after PAB ( $n=5-11$ per group). (I) Long-term overall survival curve of Slit3 $\%$ and WT mice from day 1 after PAB. Survival analysis was performed using the Kaplan-Meier method. Log-rank test, $P=0.0494$ ( $n=18-19$ per group). Data are presented as mean $\pm \mathrm{SD}$. ${ }^{*} P<0.05,{ }^{* *} P<0.01$, and ${ }^{* * *} P<0.001$ vs. WT mice using the unpaired 2-tailed Student's $t$ test (B, E, and $\left.\mathbf{F}\right), 1$-way ANOVA with Tamhane T2 multiple comparisons test (G), or 2-way ANOVA with Tukey's multiple comparisons test (C and $\mathbf{D})$. BSL, 7- to 9-week-old baseline mice before surgery.

end-diastolic posterior wall thickness, mass, end-diastolic volume, and end-systolic volume at 3, 7, and 16 weeks (Supplemental Figure 4E). In agreement with these observations, expression of hypertrophy-related genes (Nppb, Nppa, and Myh7) was also reduced in Slit $3^{-1-}$ mice (Figure 4D and Supplemental Figure 4D). Further, the LV function was preserved at 7 and 16 weeks in Slit $3^{-/-}$mice after TAC (Figure $4 \mathrm{~F}$ and Supplemental Figure 4B). Most importantly, the long-term survival was significantly enhanced in Slit3-1mice where null mice remained viable over a 1-year time course compared with more than $60 \%$ mortality in the control group (Figure $4 \mathrm{G}$ ).

SLIT3 deficiency attenuates $R V$ fibrosis and adverse remodeling. Next, we subjected animals to PAB to determine the importance of SLIT3 in RV pressure overload. The effects of SLIT3 deficiency were even more apparent in blunting the adverse remodeling response in the context of RV pressure overload, a hemodynamic condition found in congenital heart disease that can lead to RV failure and has no effective medical treatment besides heart transplantation (40). The RV free wall fibrosis area, cardiomyocyte 
A

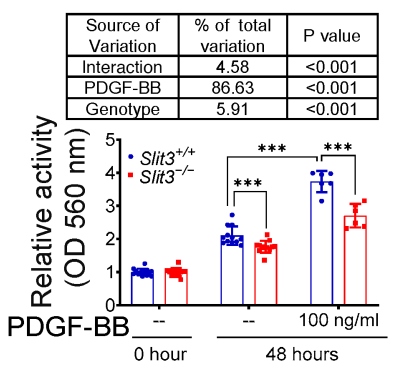

B

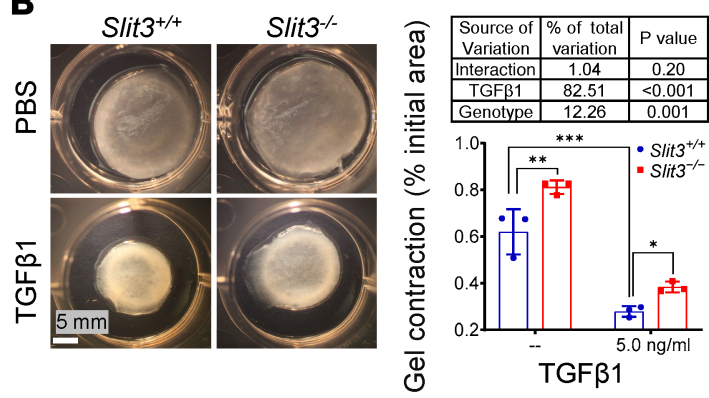

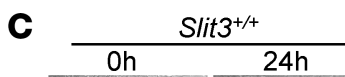

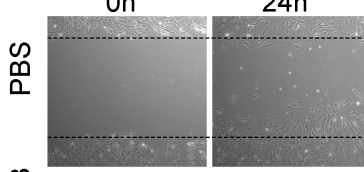

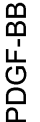

○

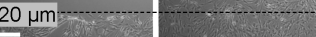

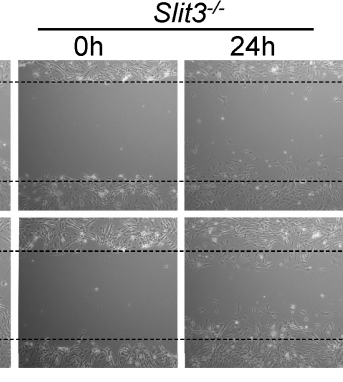

Slit3 ${ }^{-/-}$

D

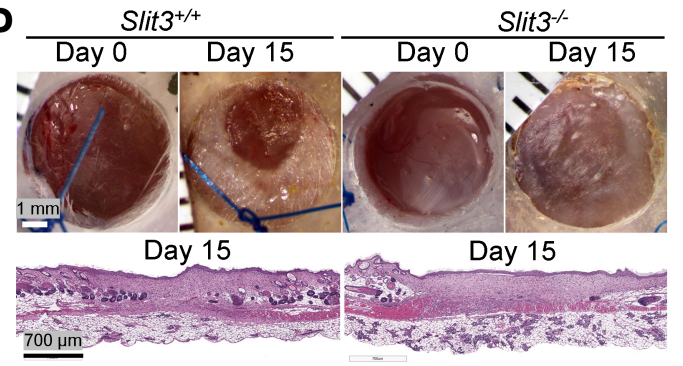

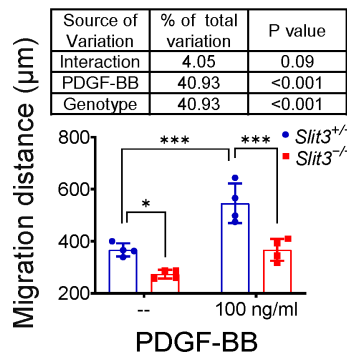

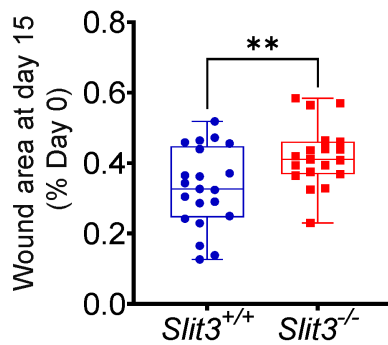

Figure 6. SLIT3 deficiency inhibits fibroblast biological activity. (A) MTT cell proliferation assay. Slit3 ${ }^{-/-}$and WT aortic adventitial fibroblasts were seeded in 96-well plates (10\% FBS medium) and treated with PBS or PDCF-BB (100 ng/mL). Absorbance was measured at $560 \mathrm{~nm}$ at 0 and 48 hours after treatments ( $n=6-14$ per group). (B) Floating collagen gel contraction assay. Representative images and area quantification of collagen gel. Slit3 ${ }^{-/-}$and WT lung fibroblasts were seeded in PBS or TGF- $\beta 1(5.0 \mathrm{ng} / \mathrm{mL})$ added to collagen gel $(1 \mathrm{mg} / \mathrm{mL}$ collagen type I, $0.5 \%$ FBS). The final to initial area ratio was determined at 24 hours after floating ( $n=3$ per group). Scale bars: $5 \mathrm{~mm}$. (C) Scratch wound healing assay. Representative images and migration distance quantification of scratch wound healing. Slit3 ${ }^{-/}$and WT lung fibroblasts were seeded in 6-well plates (1\% FBS medium) and treated with PBS or PDCF-BB (100 ng/mL). The migration distance was determined at 24 hours after scratching $(n=4)$. Scale bars: $20 \mu \mathrm{m}$. (D) Excisional wound healing assay. Representative gross and histological images (hematoxylin and eosin staining) and quantification of wound area in 8-week-old Slit3 ${ }^{--}$and WT mice at the day of surgery (day 0 ) and 15 days (day 15$)$ after surgery ( $n=19-21$ per group). Scale bars: 1 mm and $700 \mu \mathrm{m}$ from top to bottom. Data are presented as mean \pm SD. In vitro experiments were performed at least 3 times independently. ${ }^{*} P<0.05,{ }^{* *} P<0.01$, and ${ }^{* * *} P<0.001$ vs. WT mice or cells using the unpaired 2-tailed Student's $t$ test (D) or 2-way ANOVA with 2-stage step-up method of Benjamini, Krieger, and Yekutieli multiple comparisons test (A-C).

cross-sectional area, end-diastolic diameter and area, RV weight to LV plus septum weight ratio, RV/LV end-systolic diameter ratio (Figure 5, A-D; and Supplemental Figure 5, B and D), and transcript levels of Col1a1, Nppb, Col3a1, Nppa, and Myh7 (Figure 5F and Supplemental Figure 5F) were all reduced in Slit $^{-1-}$ mice at 2 and 4 weeks, together indicating that knockout animals had a decreased adverse fibrotic and hypertrophic response. In addition, after PAB, Slit3 $3^{-1-}$ mice had preserved RV FS at 2 weeks, reduced liver congestion at 4 weeks, and markedly increased survival (Figure 5, H and I; and Supplemental Figure 5, C and E).

Interestingly, the transcript levels of Slit3, as well as those of Colla1 and Col3a1, were all increased significantly at 1-2 weeks, then returned to baseline levels in WT mice after TAC and PAB (Supplemental Figure 3, B and D; and ref. 41). Similarly, the peak aortic gradients in post-TAC WT mice increased significantly from day 3 to day 21 , as described previously and attributed to the development 
A Adventitial fibroblasts
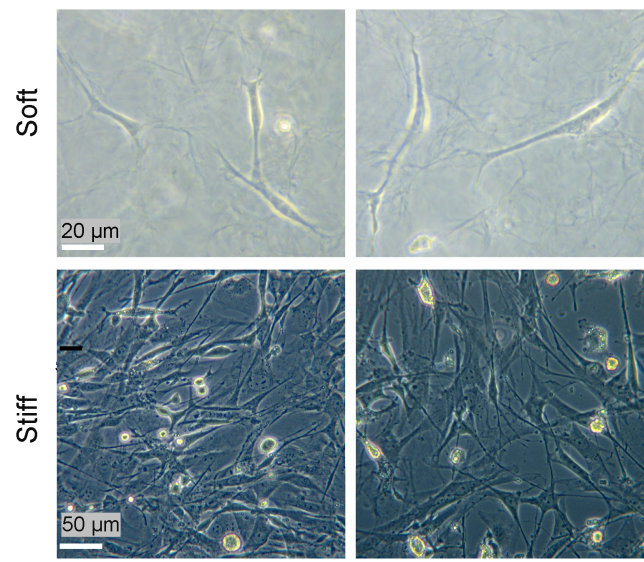

D

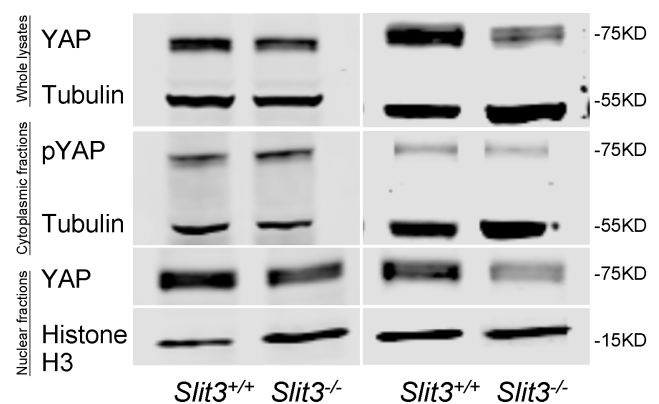

Lung fibroblasts
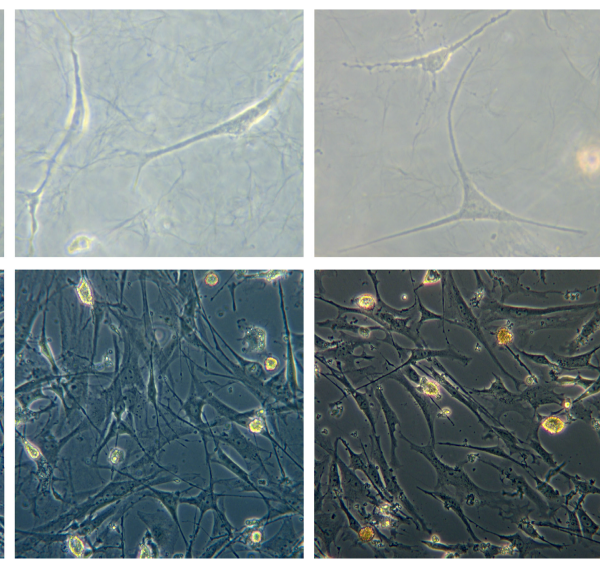

B

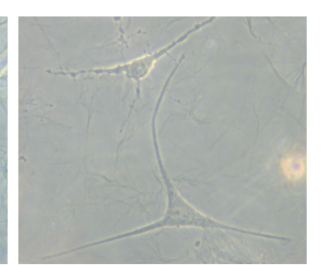

E

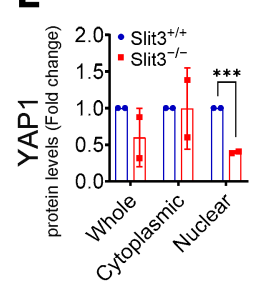

H

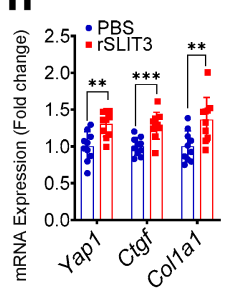

F
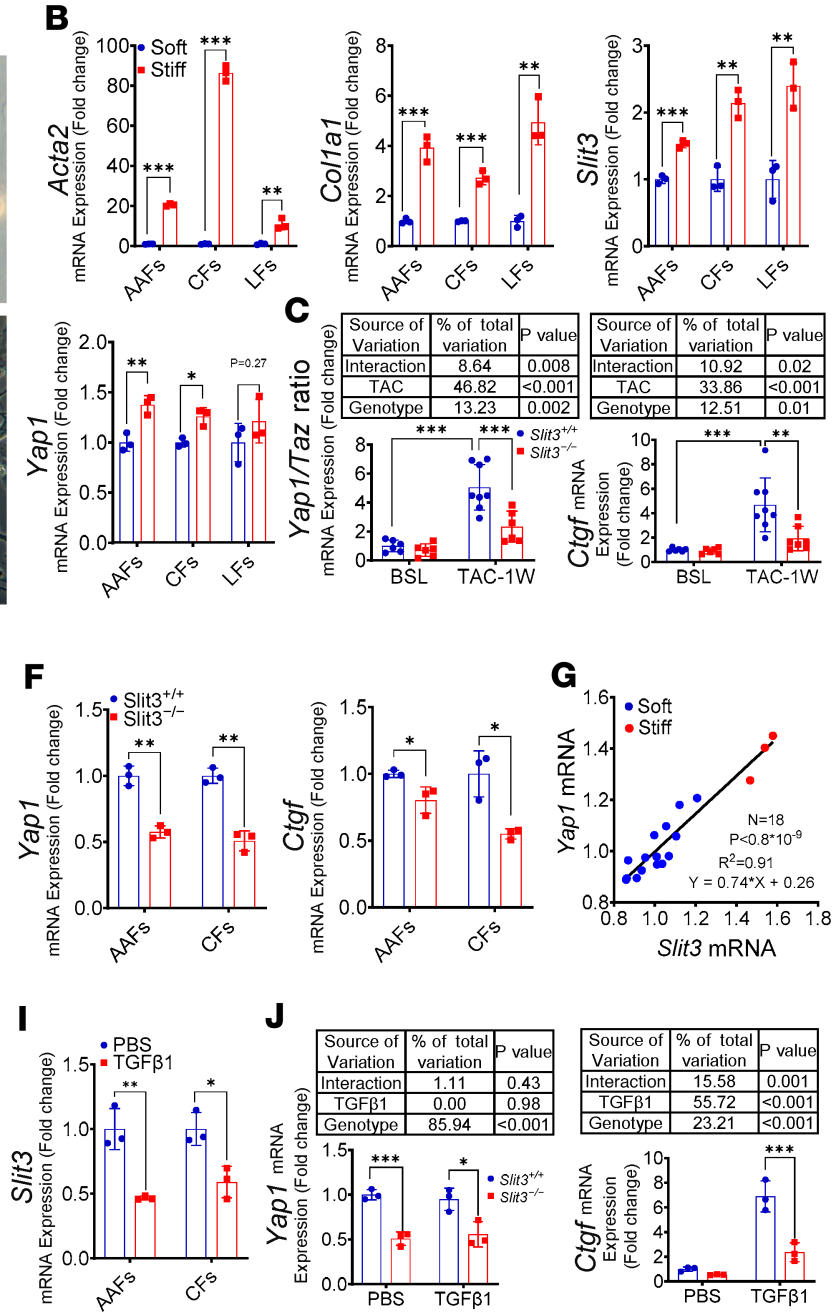

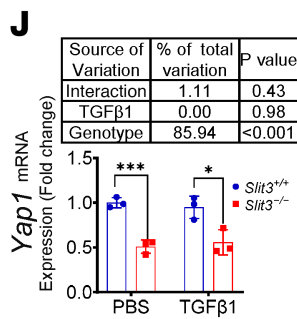

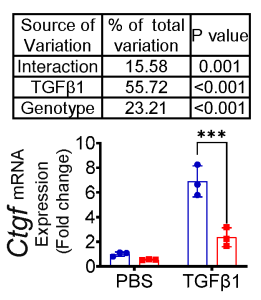

Figure 7. SLIT3 regulates YAP1 and fibrillar collagen production. (A and B) Representative images and transcript levels of Acta2, Col1a1, Slit3, and Yap1 in adult primary fibroblasts cultured on stiff surfaces (6-well plastic tissue culture plate, $10 \% \mathrm{FBS}$ ) or in soft collagen gel ( $1 \mathrm{mg} / \mathrm{mL}$ collagen type I, $1 \% \mathrm{FBS}$ ) for 24 hours. From left to right, aortic adventitial fibroblasts (AAFs), cardiac fibroblasts (CFs), and lung fibroblasts (LFs) ( $n=3$ per group). Scale bars: $20 \mu \mathrm{m}$ and $50 \mu \mathrm{m}$ from top to bottom. (C) Transcript levels of Yap1/Taz ratio and Ctgf in the left ventricle in Slit3 $3^{-/}$and WT mice before and at 1 week after TAC ( $n=6-8$ per group). (D and E) Western blots of YAP1 and phospho-YAP1 in Slit3 ${ }^{--}$and WT CFs and LFs cultured on a stiff surface (6-well plastic tissue culture plate) with quantification $(n=2)$. (F) Transcript levels of Yap1 and Ctgf in Slit3 ${ }^{--}$and WT AAFs and CFs in soft collagen gel $(1 \mathrm{mg} / \mathrm{mL}$ collagen type I, $1 \%$ FBS) for 24 hours ( $n=3$ per group). (G) Linear regression analysis between the transcript levels of Slit3 and Yap1 in WT AAFs cultured on stiff surfaces (6-well plastic tissue culture plate, 10\% FBS) or in soft collagen gel (1 mg/mL collagen type I, 1\% FBS) for 24 hours $\left(R^{2}=0.9114, n=18\right)$. (H) Transcript levels of Yap1, Ctgf, and Col1a1 in Slit3 ${ }^{-1-}$ LFs cultured in PBS or recombinant SLIT3 (rSLIT3, aa 34-1116, $1 \mu \mathrm{g} / \mathrm{mL}$ ) added to collagen gel (1 mg/mL collagen type I, $0.5 \%$ FBS) for 24 hours ( $n=10$ per group). (I) Transcript levels of Slit3 in WT AAFs and CFs cultured in PBS or TCF- $\beta 1(5.0 \mathrm{ng} / \mathrm{mL})$ added to collagen gel $(1 \mathrm{mg} / \mathrm{mL}$ collagen type I, $0.5 \%$ FBS) for 24 hours ( $n=3$ per group). (J) Transcript levels of Yap1 and Ctgf in Slit3 $\%$ and WT CFs cultured in PBS or TCF- $\beta 1$ (5.0 ng/mL) added collagen gel $(1 \mathrm{mg} / \mathrm{mL}$ collagen type I, $0.5 \% \mathrm{FBS})$ for 24 hours ( $n=3$ per group). Data are presented as mean $\pm \mathrm{SD}$. In vitro experiments were independently performed at least 3 times unless indicated otherwise. ${ }^{*} P<0.05$, ${ }^{* *} P<0.01$, and ${ }^{* * *} P<0.001$ vs. WT mice or cells using the unpaired 2-tailed Student's $t$ test (B, E, F, $\mathbf{H}$, and $\mathbf{I}$ ) and 2-way ANOVA with Tukey's multiple comparisons test ( $\mathbf{C}$ and $\mathbf{J})$.

of compensatory LV hypertrophy and the recovery of peak systolic wall stress index (42), then returned to initial levels after day 42 with the transition from compensated to decompensated heart failure as indicated by the deterioration of LV function (Supplemental Figure 3A). By contrast, in Slit3 $3^{-/}$mice, peak-to-baseline ratios of fibrosis-related gene transcript levels were all decreased after TAC and PAB, and changes in peak gradients and LV function were both relatively mild throughout the study period without a time-dependent linear trend after TAC (Supplemental Figure 3C). Although Colla1 and Col3a1 transcript levels were significantly different between baseline WT and Slit $3^{-/-}$mice, there was a genotypic effect on the transcription of these fibrillar collagen genes in response to pressure overload surgery, and Slit3 ${ }^{-1-}$ mice had a weaker fibrotic response to TAC and PAB than WT mice (2-way ANOVA, $P<0.05$; Supplemental Figure 3, E and F). 
SLIT3 deficiency inhibits fibroblast activity. Growth factors such as transforming growth factor- $\beta 1$ (TGF- $\beta 1$ ) and platelet-derived growth factor BB (PDGF-BB) are overexpressed and act as important mediators of fibroblast activation in the pathogenesis of cardiac fibrotic and hypertrophic remodeling $(8,43-45)$. Therefore, TGF- $\beta 1$ and PDGF-BB were used to activate primary adult fibroblasts in vitro. Compared with passage-matched WT cells, Slit $3^{-1-}$ fibroblasts treated with or without either PDGF-BB or TGF- $\beta 1$ displayed significantly inhibited proliferation, migration, and contraction activities (Figure 6, A-C). Twoway ANOVA demonstrated significant interaction between SLIT3 and PDGF-BB in fibroblast proliferation assays, although it accounted for only $4.5 \%$ of all variation. No other significant interactions were observed in the contraction and migration assays. In the proliferation and contraction assays, PDGF-BB and TGF- $\beta 1$ each contributed more than $82 \%$ of the total variation, whereas the genotypic effect of Slit 3 presence or absence in the fibroblasts accounted for only $5.9 \%$ or $12.3 \%$, respectively (Figure 6, A and B). Overall, these functional results indicated that SLIT3's stimulation of fibroblast activity appears to be relatively independent of recombinant TGF- $\beta 1$ for the concentrations of the factors that we used in our experiments. To further confirm the effect of SLIT3 on fibroblast biological activity in vivo outside the heart, a splinted skin excisional wound model was performed (46), and consistently, we found that dermal wound healing, which is critically dependent upon fibroblast function and fibrillar collagen production (47), was also delayed in Slit $3^{-1-}$ mice (Figure 6D). In addition, the expression of periostin, a marker of fibroblast activation (48), was decreased in the hearts of Slit $3^{-1-}$ mice that had undergone TAC (Supplemental Figure 9).

SLIT3 regulates YAP1 and fibrillar collagen production. Fibrillar collagen contributes to the biomechanical properties of connective tissue (21), where resident fibroblasts are commonly quiescent (49) and protected from persistent external loads by dynamically regulated ECM in vivo (50). Resident cardiac fibroblasts are also quiescent cells with a low proliferation and ECM production under normal hemodynamic conditions (51) and are the principal source of activated fibroblasts in injured mammalian hearts $(52,53)$. We observed that acutely increasing ventricular wall stress with TAC or PAB resulted in the activation of resident fibroblasts and increase of myocardial production of fibrous collagen and SLIT3 (Figure 4E, Figure 5G, and ref. 54). The mechanical microenvironment is known to be an important determinant of fibroblast activation in vitro and in vivo (55). To further investigate the effect of the mechanical microenvironment on the role of SLIT3-induced fibroblast activation in vitro, aortic adventitial fibroblasts (AAFs), cardiac fibroblasts (CFs), and lung fibroblasts (LFs) were cultured on stiff plastic tissue surfaces or in soft collagen type I gel, as fibroblasts adaptate their internal stiffness to match that of their substrate (56), and local stiffness acts as an important mechanical effector (57). Importantly, we found that transcript levels of Acta2, Colla1, Slit3, and Yap1 were all significantly increased by culturing fibroblasts on a stiff surface (Figure 7, A and B), indicating that SLIT3 transcription may be regulated by the surrounding mechanical microenvironment.

Because the transcription factors yes-associated protein 1 and tafazzin (YAP1/TAZ) have been identified as important mediators of mechanotransduction signals in fibroblasts and widely regulate fibroblast biological activities, including proliferation, migration, and fibrillar collagen production (58-61), the potential association of SLIT3 and YAP1/TAZ were examined. We found that the transcript levels of Yap1, rather than Taz, and its regulated gene Ctgf in the cardiac ventricles after TAC were reduced significantly in Slit $3^{-1-}$ compared with WT mice (Figure 7C, Supplemental Figure 6B, and ref. 62). In vitro, both the transcript levels of Yap1 and Ctgf, as well as the nuclear translocation of YAP1, were reduced significantly in Slit3 $3^{-1}$ as compared with WT fibroblasts (Figure 7, D-F; see full, uncut gels in the online supplemental material). Further, there was a strong linear association between the transcript levels of Slit3 and Yap1 of fibroblasts cultured on both soft and stiff surfaces $\left(R^{2}=0.91\right.$, Figure 7G), and recombinant SLIT3 (rSLIT3, aa 34-1116) treatment significantly increased the transcript levels of Yap1 as well as its downstream gene targets Ctgf and Colla1 in fibroblasts (ref. 63 and Figure 7H), suggesting that SLIT3 has a positive regulatory effect on Yap1 transcription. In addition, although TGF- $\beta 1$ treatment of fibroblasts significantly reduced the transcript levels of Slit3, there was a genotypic effect on Ctgf expression response to TGF- $\beta 1$; i.e., Slit $3^{-1-}$ fibroblasts had a blunted response to TGF- $\beta 1$ as compared with WT fibroblasts (2-way ANOVA, $P<0.001$, Figure 7 , I and J).

\section{Discussion}

Since its original description as an axon guidance molecule (64), there has been mounting evidence that SLIT3, a large secreted glycoprotein (65), has other important extraneuronal functions (14, 16, 17, 66-69). Here we provide evidence that SLIT3 plays an important role in the function of fibroblasts, which express ROBO1 (Figure 1D), also the principal Roundabout receptor for SLIT3-mediated osteogenesis $(14,18)$, and consequently, plays a role 
in regulating the content of fibrillar collagen in the heart during conditions of homeostasis as well as stress. Furthermore, our results demonstrate that SLIT3 also regulates fibroblasts and the collagen content in extracardiac tissues, underscoring SLIT3's importance in collagen-rich tissues and, in turn, their biomechanical properties.

Similar to previous reports, outbred SLIT3 deficiency mice have reduced bone mass (Figure 3I). Interestingly, for this phenotype, Xu et al. (14) and Kim et al. (18) reported contradictory mechanisms and focused mainly on the effective sources of SLIT3. However, using multiple lines of evidence, Li et al. (70) recently demonstrated that skeletal SLIT3 is secreted mainly by osteoblasts rather than osteoclasts. Osteoblasts are differentiated from mesenchymal stromal cells and have been regarded as sophisticated fibroblasts (71). It is possible that osteoporosis $(14,18)$, congenital $\mathrm{CDH}(13,17)$, and membranous ventricular septal defects $(22,23)$, all observed in SLIT3-deficient animals, are the organ-specific manifestation of the systemic collagen reduction caused by SLIT3 deficiency, as fibrillar collagen type I is the main and functional component of all of these tissues.

By inhibiting the biosynthesis of fibrillar collagen, targeting SLIT3 may be counterproductive in the setting of osteoporosis $(14,18)$, bone fracture (14), wound healing (Figure 6D), and after MI (Figure 3H); however, it may have beneficial effects in other contexts, such as pressure overload adverse remodeling of the ventricle. After TAC or $\mathrm{PAB}$, ventricular pressure overload increases peak wall stress and induces reactive tissue remodeling that is thought to occur as a compensatory response to normalize wall stress $(42,72)$. Within 3 weeks after TAC, the aortic peak gradient was positively correlated with myocardial hypertrophy (Supplemental Figure $3 \mathrm{G}$ ), and correspondingly, the early-stage remodeling was compensatory in both WT and Slit $3^{-1-}$ mice (Figure $4 \mathrm{~F}$ and ref. 42). Notably, different degrees of reactive remodeling induced by similar initial hemodynamic load were observed in WT and Slit3 ${ }^{-1-}$ post-TAC mice (72), as blunted fibrillar collagen synthesis together with a stable aortic gradient may have permitted the recovery of LV wall stress in the absence of excessive hypertrophy in Slit $3^{-1-}$ mice. Furthermore, suppressing reactive remodeling by SLIT3 deficiency yielded a long-term cardioprotective effect in the setting of persistent afterload stress in LV, as the response in WT mice was compensatory but also excessive and ultimately maladaptive (Figure 4, F and G; and refs. 73, 74).

Unlike the more robust and pressure-resistant left ventricle (75), pressure overload of the right ventricle induced by $\mathrm{PAB}$ resulted in stronger reactive remolding, including marked free wall fibrosis, which is an important pathophysiological factor correlating with disease severity (76-78) and promotes diastolic dysfunction by reducing myocardial compliance $(7,79,80)$. Perhaps preserving RV compliance and diastolic function by reducing collagen accumulation induced by PAB, SLIT3 deficiency attenuated its dilation (Figure 5, C and D), prevented the transition from adaptive concentric remodeling to maladaptive hypertrophy and fibrosis associated with decompensated failure (Supplemental Figure 5, B and C), and, importantly, exhibited an excellent long-term cardioprotective effect in the setting of persistent afterload (Figure 5, H and I; and refs. 7, 81, 82). Interestingly, compared with other forms of pulmonary arterial hypertension, the right ventricles of patients with Eisenmenger's syndrome exhibit significantly less fibrosis and are better adapted to increased pressure overload (83).

Several preclinical studies have demonstrated that inhibiting load-induced myocardial hypertrophy might be beneficial in the short term despite persistence of the initiating stimulus $(54,84-88)$. It is also evident that excessive inhibition of the required compensatory response to normalize increased wall stress is associated with cardiomyocyte damage, depressed systolic function, and increased mortality $(42,72,89$, 90). This did not seem to be the case in SLIT3-deficient animals undergoing TAC, as only 3 of the total of $13(23 \%)$ animals demonstrated localized fibrosis possibly occurring after cardiomyocyte apoptosis or necrosis (91) at the junctional area of the posterior ventricular septum and free wall and 1 animal $(0.08 \%)$ suffered from transient cardiac dysfunction that recovered within 15 days while, in general, the cardiac function of Slit3 ${ }^{-/-}$mice was well preserved after TAC (Supplemental Figure 7, A-F). These findings indicate that the inhibitory effect of SLIT3 deficiency on the hypertrophy response induced by pressure overload is moderate and may be secondary to defective fibroblast/cardiomyocyte crosstalk, although a direct effect of SLIT3 on cardiomyocytes is also possible (87, 92-94).

On the premise of normalizing wall stress and preventing myocyte death, our results indicate that minimizing, but not completely abrogating, the reactive remodeling, especially fibrosis induced by pressure overload, is a promising strategy to pursue therapeutic long-term cardioprotective effects in both the left ventricle and right ventricle. Our results indicate that global SLIT3 deficiency appears to yield such a beneficial effect, achieving the "Goldilocks Zone" of tempering hypertrophy and fibrosis but not completely abolishing them. While SLIT3 is expressed mainly in fibroblast and other connective tissue 
cell lineages, additional work with lineage-specific and inducible-knockout animals is needed to confirm that fibroblast-mediated SLIT3 is responsible for the phenotype that we observed in our global-knockout animals. Nonetheless, from a therapeutics and translational perspective, targeting SLIT3 and its downstream signals via, for example, small-molecule antagonists, will likely target most cells indiscriminately and thus may reflect the situation in our global knockouts with the favorable phenotype in the setting of ventricular pressure overload.

It has been previously shown that pressure overload by TAC induces an acute increase in ventricular wall stress that is later normalized within 10 days because of remodeling (42), which is nearly contemporaneous with the peak expression of Slit3 (Figure 4E and Figure 5G) and Col1a1 (Supplemental Figure $3, \mathrm{~B}$ and D) that we observed in our model (41). Interestingly, the signaling activity of TGF- $\beta 1$, known to play an important role in fibrosis $(95,96)$, has been demonstrated to start and progressively increase up to at least 9 weeks after TAC (97). These results, taken together with our in vitro findings that the mechanical microenvironment can stimulate Slit3 transcription, suggest that pressure overload may provide a mechanical stimulus for Slit3 transcription. Further supporting a role for SLIT3 in mechanotransduction is our finding that fibroblasts from SLIT3-deficient animals had decreased nuclear and total YAP1 content, as well as the decreased transcription of TEAD target gene $C \operatorname{tg} f$ (Figure 7, D-F). In addition, exposing SLIT3-deficient fibroblasts to a ROBO1-binding rSLIT3 N-terminal fragment could acutely stimulate Yap1 and Ctgf transcription (Figure 7H), indicating that SLIT3 deficiency attenuated the effect of mechanical stimulation on fibroblasts by downregulating the transcript levels of Yap1, an important sensor and mediator of mechanical cues (Graphical Abstract and ref. 60).

The findings of this study are especially relevant to children with congenital heart disease or pulmonary hypertension who are chronically exposed to either left or right ventricular pressure overload and whose outcomes are dependent upon the maintenance of ventricular function. Importantly, children with pressure overload of the right ventricle have no effective medical treatment beyond heart transplantation $(1,6)$, and thus targeting SLIT3 or fibroblast-specific YAP1 activation $(59,98,99)$, rather than cardiomyocyte-specific YAP1 activation (100), may a promising strategy to inhibit adverse remodeling and preserve ventricular function by reducing fibrosis (101). Furthermore, $\mathrm{MI}$ is rare in children, and hence myocardial rupture after infarction as a potential side effect of SLIT3 inhibition would be unusual in children with ventricular pressure overload. In sum, the results of this study identify SLIT3 as a potential therapeutic target to prevent adverse remodeling and to preserve ventricular function in the setting of chronic ventricular pressure overload.

\section{Methods}

FANTOM5 data processing. Expression levels of the genes of interest in primary cell types with replicates were downloaded from the FANTOM5 database (119 cell types). Analysis was performed as described by Schafer et al. (102). Briefly, the gene expression level was obtained by first adding all counts that were assigned to a specific gene and then normalized by the library size to derive the TPM for each gene. We determined the TPM for our genes of interest in 512 primary cell samples that included cell types from all lineages.

Slit $3^{-1-}$ (knockout) mice. Slit $3^{-1-}$ mice on a CD-1 background were obtained from Sean McLean, University of North Carolina, Chapel Hill, North Carolina, USA (103). WT CD-1 mice were purchased from Charles River Laboratories. Similar to the inbred mice (13), for the genotyping analysis of CD-1 WT and Slit $3^{-/-}$mice, 3 primers were used. The WT allele amplified a 250-bp fragment (primers a and b), whereas the null allele amplified a 410-bp fragment (primers a and c). PCR conditions were 35 cycles at $94^{\circ} \mathrm{C}$ for 45 seconds, $60^{\circ} \mathrm{C}$ for 45 seconds, and $72^{\circ} \mathrm{C}$ for 45 seconds. The oligonucleotide sequences used for genotyping were primer a ( 5 GCGCCTCCTCGGGCTCCTCGTGTC 3', sense), primer b (5' TGCGGGGGATGCCCCGAGGAA 3', antisense), and primer c (5' CGGATTCTCCGTGGGAACAAACGG 3', antisense).

Mouse models. Mouse cardiac procedures, including TAC, PAB, and left anterior descending coronary artery ligation, were performed as previously described with the aid of an operating microscope (104). Details of these procedures are provided in the Supplemental Methods.

The mouse excisional wound splinting model was performed as previously described (46). After hair removal, the dorsal skin of the chest was folded and punched with a 5-mm-diameter biopsy punch to create 2 full-thickness excisional wounds besides the midline. Donut-like splints with a 6-mm-diameter hole were glued and sutured to the skin around the wound. Digital photographs of individual wounds were taken on days 0 and 15 after surgery. 
Mouse left ventricle toughness. After anesthesia with inhalational isoflurane, a sternotomy was performed, and the ascending aorta was clamped. The heart was removed, and the left ventricle was perfused with $4^{\circ} \mathrm{C}$ histidine-tryptophan-ketoglutarate solution to obtain arrest in diastole. A balloon-tipped catheter was inserted into the left ventricle through the mitral valve, fixed by a purse-string suture, and gradually inflated until LV rupture. The heart was then removed and weighed. Passive inflation was accomplished with a Gilson MINIPULS 3 pump using a constant rate. During passive inflation of the left ventricle, pressure was monitored with a pressure transducer (ADInstruments MLT0670), and data were acquired with a PowerLab DAQ device and recorded with LabChart software. Pressure-volume (PV) curves were then generated, and the area under the PV curves after subtracting the PV curve obtained with the balloon only was calculated as the energy of LV rupture, which was then normalized to the heart mass to obtain the energy density.

Echocardiography. Transthoracic echocardiography was performed by the University of Michigan Cardiovascular Phenotyping Core, as described previously (105) and in the Supplemental Methods.

Primary murine cell isolation and culture. Cells were isolated from 6- to 8-week-old CD-1 WT and Slit $3^{-1-}$ mice. CFs and cardiomyocytes (106), LFs (107), as well as AAFs and vascular smooth muscle cells (108) were isolated and cultured as described in the Supplemental Methods.

Recombinant proteins. Human TGF- $\beta 1$ (240-B, R\&D Systems, Bio-Techne), rat PDGF-BB (520-BB/CF, R\&D Systems, Bio-Techne), human FGF-basic (03-0002, STEMGENT), and mouse Slit3 (aa 34-1116, 9296-SL-050, R\&D Systems, Bio-Techne) were used at the indicated concentrations.

Cellular proliferation and functional assays. Cell proliferation, scratch wound and migration, and floating collagen gel contraction are detailed in the Supplemental Methods.

Tissue sample preparation and hydroxyproline assay. Mice were euthanized by cervical dislocation under anesthesia with $2 \%$ isoflurane. Next, the chest, abdomen, and right atrium were opened, and the left ventricle was perfused with $20 \mathrm{~mL} 4^{\circ} \mathrm{C}$ PBS to flush the blood from the vessels. The heart, left lung, spleen, left kidney, aorta, dorsal skin, and right femur were sequentially harvested and stored at $4^{\circ} \mathrm{C}$ in HBSS. Then any attached adjacent tissues were removed from the organs with the aid of an operating microscope. For the hydroxyproline assay, tissues were weighed (80-90 mg was used) and placed into pressure-tight, 2.0-mL, screw-top, polypropylene tubes. For real-time qPCR, 5-10 mg tissues were placed into $1.5-\mathrm{mL}$ tubes. All samples were then immediately stored at $-80^{\circ} \mathrm{C}$. The hydroxyproline assay kit (MilliporeSigma, catalog MAK008) was used to quantify the total tissue collagen content according to the manufacturer's protocol. Briefly, $6 \mathrm{M} \mathrm{HCl}$ was added to the $2.0-\mathrm{mL}$, screw-top, polypropylene tubes as $20 \mu \mathrm{L}$ per $1 \mathrm{mg}$ tissue, and these samples were hydrolyzed for 24 hours under a tight lid at $110^{\circ} \mathrm{C}$. Next, the tubes were mixed and centrifuged at 10,000 $\mathrm{g}$ for 3 minutes. Afterward, $10 \mu \mathrm{L}$ supernatant from the tubes of bone and skin was diluted with $190 \mu \mathrm{L}$ water in new 1.5 -mL tubes. Then $20 \mu \mathrm{L}$ (heart, kidney, and spleen), $10 \mu \mathrm{L}$ (lung), and $20 \mu \mathrm{L}$ diluted (bone and skin) supernatants were transferred to 96-well plates, respectively. The 96 -well plates were placed in a $60^{\circ} \mathrm{C}$ oven to dry samples. Then $100 \mu \mathrm{L}$ Chloramine T/Oxidation buffer mixture (part of the kit) was added to each reaction well and standard well. The plates were incubated at $25^{\circ} \mathrm{C}$ for 10 minutes. Then $100 \mu \mathrm{L}$ Diluted DMAB Reagent (MilliporeSigma) was added to each reaction well and standard well, and then the plates were incubated in a $60^{\circ} \mathrm{C}$ oven for 90 minutes. The absorbance of each well was measured at $560 \mathrm{~nm}$ with a spectrophotometer.

Real-time quantitative PCR gene transcript level analysis. Total RNA was extracted from frozen tissues stored at $-80^{\circ} \mathrm{C}$ or cells cultured in plates using RNeasy Mini Kit (catalog 74106, QIAGEN). About 0.5-1 $\mu$ g of total RNA was used to synthesize the cDNA with the cDNA Synthesis Kit (lot 028755, Quanta bio) and according to the manufacturer's protocol. Real-time quantitative PCR (RT qPCR) gene transcript level analysis was performed on duplicate samples with SYBR Green (lot 028416, Quanta bio) technology using a StepOnePlus (Applied Biosystems, Thermo Fisher Scientific). The amplification protocol consisted of 40 cycles, including denaturation at $95^{\circ} \mathrm{C}$ for 15 seconds, annealing, and extension at $60^{\circ} \mathrm{C}$ for 60 seconds. The gene expression levels were normalized to that of the housekeeping gene GAPDH. The $2^{-\Delta \Delta C t}$ method was used to calculate the fold change. Sequences of the RT qPCR primer pairs are provided in the Supplemental Methods.

Western blot. Total protein was extracted from cultured cells in RIPA buffer supplemented with EDTA-free Halt protease inhibitor cocktail (Thermo Fisher Scientific). Cytoplasmic and nuclear proteins were extracted using NE-PER Nuclear and Cytoplasmic Extraction Reagents (Thermo Fisher Scientific) according to the manufacturer's instructions. Equal amounts of protein were loaded onto a precast NuPAGE $4 \%$ to $12 \%$ Bis-Tris mini protein gel (Invitrogen, Thermo Fisher Scientific) and transferred to a nitrocellulose membrane by Trans-Blot Turbo Transfer System (Bio-Rad). Transferred membranes were blocked for 1 hour in TBS containing $5 \%$ nonfat dried milk followed 
by overnight incubation at $4^{\circ} \mathrm{C}$ in the corresponding primary antibodies. The following antibodies purchased from Cell Signaling Technology were used at 1:1000 dilution: $\beta$-tubulin (catalog 2146S), histone H3 (catalog 14269S), YAP (catalog 12395S), and phospho-YAP (Ser127; catalog 4911S). After 3 washes for 5 minutes in $1 \times$ Tris-Buffered Saline, 0.1\% TWEEN 20 Detergent wash buffer (MilliporeSigma), following incubation with goat anti-mouse IgG $(\mathrm{H}+\mathrm{L})$ Highly Cross-Adsorbed Secondary Antibody, Alexa Fluor 680 (catalog A-21058); and goat anti-rabbit IgG $(\mathrm{H}+\mathrm{L})$ Highly Cross-Adsorbed Secondary Antibody, Alexa Fluor Plus 800 (catalog A32735; 1:5000; Thermo Fisher Scientific) and then visualized by using an Odyssey CLx imaging system (LI-COR Biosciences).

Histology. Tissues from mouse models were fixed in 10\% formalin, dehydrated and embedded in paraffin, and sectioned at $5 \mu \mathrm{m}$. Masson's trichrome staining was used to detect collagen fibers, while hematoxylin and eosin staining was used to determine cardiomyocyte cross-sectional area, skin hair follicle density, and wound healing process. The images of sections were captured, scanned, and then analyzed with Aperio Image Scope (version 12.1) and Image-Pro Plus (version 7).

Immunofluorescence/Immunohistochemistry. Cells were fixed and permeabilized in 4\% paraformaldehyde and $0.1 \%$ Triton X-100 in PBS, respectively, thenblocked with 3\% BSA in PBS for 30 minutes. Subsequently, cells were stained with primary antibodies targeting SLIT3 (SAB2104337: MilliporeSigma) or ROBO1 (ab7279: Abcam) at 1:200 in blocking solution at $4^{\circ} \mathrm{C}$ overnight. Then, cells were washed and incubated with the appropriate secondary antibody, donkey anti-rabbit IgG $(\mathrm{H}+\mathrm{L})$ Highly Cross-Adsorbed Alexa Fluor Plus 488 (catalog A32790, Invitrogen, Thermo Fisher Scientific), in the dark for 60 minutes at room temperature. Nuclei were stained with $1 \mu \mathrm{g} / \mathrm{mL}$ DAPI. Images were obtained using a Nikon A1 confocal microscope.

Heart paraffin sections were deparaffinized with xylene and rehydrated with serial dilutions of ethanol. After antigen retrieval, the sections were blocked with 5\% donkey serum/3\% BSA/0.1\% Triton X-100/PBS for 1 hour at room temperature. The following primary antibodies were used at 1:200 dilution overnight at $4^{\circ} \mathrm{C}$ : CD31 (AF3628: R\&D Systems, Bio-Techne) and collagen type I (Abcam ab21286). After washing 3 times for 5 minutes each, secondary antibody donkey anti-goat Alexa Fluor 488 and donkey anti-rabbit Alexa Fluor 564 (catalog A-11055 and A10042, respectively; Invitrogen, Thermo Fisher Scientific) were incubated with a 1:500 dilution for 1 hour at room temperature. Sections were then stained with DAPI. Images were acquired on an inverted Nikon A1 confocal microscope.

Statistics. All statistical analyses, including unpaired 2-tailed Student's $t$ test, 1-way and 2-way ANOVA with following multiple comparisons test, linear regression and trend, as well as survival curves were performed using GraphPad Prism software (version 8). Outliers were identified by GraphPad outlier calculator $(\alpha=0.05)$ and removed before analysis. $P$ values of less than 0.05 were regarded as statistically significant.

Study approval. All mouse experiments were performed according to the animal experimental guidelines issued by and with the approval of the IACUC of the University of Michigan.

\section{Author contributions}

MSS conceived the study and managed and arranged funding for the project. LG, MSS, S Weiss, SME, JAS, ALW, and SW designed the experiments. Wet lab experiments (cell culture, cell biology, molecular biology, histology, immunohistochemistry) were carried out by LG, SW, LS, BL, and XL. In vivo mouse experiments were performed by LG and LS. LV rupture testing and data analysis were performed by LS, CL, MS, JAS, ALW, and MSS. Data were analyzed by LG, LS, SW, MSS, SJW, SME, AE, YY, and DX. LG, MSS, SJW, and SW drafted the manuscript with input from the other authors.

\section{Acknowledgments}

Research reported in this publication was supported by the National Heart Lung and Blood Institute of the NIH under award number K08HL146351 (to MSS), a grant from the Children's Health Foundation (to MSS), a Frankel Cardiovascular Center McKay Award (to MSS), the Scheutz and Peace families, and the University of Michigan Department of Cardiac Surgery. We thank Kimber Converso-Baran for expert technical help with echocardiography and Sean McLean for the Slit3-knockout mice.

Address correspondence to: Ming-Sing Si, Section of Pediatric Cardiovascular Surgery, University of Michigan, 1500 East Medical Center Drive, Ann Arbor, MI 48109. USA; Email: mingsing@med.umich.edu.

LG's and YY's present address is: Department of Cardiovascular Surgery, Second Xiangya Hospital of Central South University, Changsha, China. 
LS's present address is: Department of Cardiothoracic Surgery, Shanghai Children's Medical Center, Shanghai Jiaotong University, Shanghai, China.

1. Jain A, McNamara PJ. Persistent pulmonary hypertension of the newborn: advances in diagnosis and treatment. Semin Fetal Neonatal Med. 2015;20(4):262-271.

2. Filippov AA, Del Nido PJ, Vasilyev NV. Management of systemic right ventricular failure in patients with congenitally corrected transposition of the great arteries. Circulation. 2016;134(17):1293-1302.

3. Barron DJ, Kilby MD, Davies B, Wright JG, Jones TJ, Brawn WJ. Hypoplastic left heart syndrome. Lancet. 2009;374(9689):551-564

4. Schwartz SM, Gordon D, Mosca RS, Bove EL, Heidelberger KP, Kulik TJ. Collagen content in normal, pressure, and pressure-volume overloaded developing human hearts. Am J Cardiol. 1996;77(9):734-738.

5. Ryan JJ, et al. Right ventricular adaptation and failure in pulmonary arterial hypertension. Can J Cardiol. 2015;31(4):391-406.

6. Brida M, Diller GP, Gatzoulis MA. Systemic right ventricle in adults with congenital heart disease: anatomic and phenotypic spectrum and current approach to management. Circulation. 2018;137(5):508-518.

7. Andersen S, Nielsen-Kudsk JE, Vonk Noordegraaf A, de Man FS. Right ventricular fibrosis. Circulation. 2019;139(2):269-285.

8. Travers JG, Kamal FA, Robbins J, Yutzey KE, Blaxall BC. Cardiac fibrosis: the fibroblast awakens. Circ Res. 2016;118(6):10211040 .

9. Kramer SG, Kidd T, Simpson JH, Goodman CS. Switching repulsion to attraction: changing responses to slit during transition in mesoderm migration. Science. 2001;292(5517):737-740.

10. Bagri A, et al. Slit proteins prevent midline crossing and determine the dorsoventral position of major axonal pathways in the mammalian forebrain. Neuron. 2002;33(2):233-248.

11. Plump AS, et al. Slit1 and Slit2 cooperate to prevent premature midline crossing of retinal axons in the mouse visual system. Neuron. 2002;33(2):219-232.

12. Itoh A, Miyabayashi T, Ohno M, Sakano S. Cloning and expressions of three mammalian homologues of Drosophila slit suggest possible roles for Slit in the formation and maintenance of the nervous system. Brain Res Mol Brain Res. 1998;62(2):175-186.

13. Liu J, et al. Congenital diaphragmatic hernia, kidney agenesis and cardiac defects associated with Slit3-deficiency in mice. Mech Dev. 2003;120(9):1059-1070.

14. Xu R, et al. Targeting skeletal endothelium to ameliorate bone loss. Nat Med. 2018;24(6):823-833.

15. GTEx Consortium. The Genotype-Tissue Expression (GTEx) project. Nat Genet. 2013;45(6):580-585.

16. Zhang B, Dietrich UM, Geng JG, Bicknell R, Esko JD, Wang L. Repulsive axon guidance molecule Slit3 is a novel angiogenic factor. Blood. 2009;114(19):4300-4309.

17. Yuan W, Rao Y, Babiuk RP, Greer JJ, Wu JY, Ornitz DM. A genetic model for a central (septum transversum) congenital diaphragmatic hernia in mice lacking Slit3. Proc Natl Acad Sci U S A. 2003;100(9):5217-5222.

18. Kim BJ, et al. Osteoclast-secreted SLIT3 coordinates bone resorption and formation. J Clin Invest. 2018;128(4):1429-1441.

19. Lango Allen $\mathrm{H}$, et al. Hundreds of variants clustered in genomic loci and biological pathways affect human height. Nature. 2010;467(7317):832-838.

20. Ricard-Blum S. The collagen family. Cold Spring Harb Perspect Biol. 2011;3(1):a004978.

21. Gelse K, Pöschl E, Aigner T. Collagens--structure, function, and biosynthesis. Adv Drug Deliv Rev. 2003;55(12):1531-1546.

22. Mommersteeg MT, Yeh ML, Parnavelas JG, Andrews WD. Disrupted Slit-Robo signalling results in membranous ventricular septum defects and bicuspid aortic valves. Cardiovasc Res. 2015;106(1):55-66.

23. Zhao J, Mommersteeg MTM. Slit-Robo signalling in heart development. Cardiovasc Res. 2018;114(6):794-804.

24. Fotiou E, et al. Integration of large-scale genomic data sources with evolutionary history reveals novel genetic loci for congenital heart disease. Circ Genom Precis Med. 2019;12(10):442-451.

25. Exposito JY, Valcourt U, Cluzel C, Lethias C. The fibrillar collagen family. Int J Mol Sci. 2010;11(2):407-426.

26. de Souza RR. Aging of myocardial collagen. Biogerontology. 2002;3(6):325-335.

27. Ghosh AK. Factors involved in the regulation of type I collagen gene expression: implication in fibrosis. Exp Biol Med (Maywood). 2002;227(5):301-314.

28. Noguchi S, et al. FANTOM5 CAGE profiles of human and mouse samples. Sci Data. 2017;4:170112

29. Tabula Muris Consortium, et al. Single-cell transcriptomics of 20 mouse organs creates a Tabula Muris. Nature. 2018;562(7727):367-372.

30. Alberts B, et al. Fibroblasts and their transformations: the connective-tissue cell family. In: Molecular Biology of the Cell. 4th ed. New York, New York, USA: Garland Science; 2002.

31. Domyan ET, et al. Roundabout receptors are critical for foregut separation from the body wall. Dev Cell. 2013;24(1):52-63.

32. Friedberg MK, Redington AN. Right versus left ventricular failure: differences, similarities, and interactions. Circulation. 2014;129(9):1033-1044.

33. Manabe I, Shindo T, Nagai R. Gene expression in fibroblasts and fibrosis: involvement in cardiac hypertrophy. Circ Res. 2002;91(12):1103-1113.

34. Furtado MB, Nim HT, Boyd SE, Rosenthal NA. View from the heart: cardiac fibroblasts in development, scarring and regeneration. Development. 2016;143(3):387-397.

35. Zhang $\mathrm{H}$, et al. Generation of quiescent cardiac fibroblasts from human induced pluripotent stem cells for in vitro modeling of cardiac fibrosis. Circ Res. 2019;125(5):552-566.

36. Moore-Morris T, Cattaneo P, Puceat M, Evans SM. Origins of cardiac fibroblasts. J Mol Cell Cardiol. 2016;91:1-5.

37. Schwarz ER, Dashti R. The clinical quandary of left and right ventricular diastolic dysfunction and diastolic heart failure. Cardiovasc $J$ Afr. 2010;21(4):212-220.

38. Fang L, et al. Higher levels of collagen and facilitated healing protect against ventricular rupture following myocardial infarction. Clin Sci. 2008;115(3):99-106.

39. Sane DC, Mozingo WS, Becker RC. Cardiac rupture after myocardial infarction: new insights from murine models. Cardiol Rev. 
2009;17(6):293-299.

40. Si MS, Ohye RG. Stem cell therapy for the systemic right ventricle. Expert Rev Cardiovasc Ther. 2017;15(11):813-823.

41. van den Bosch BJ, et al. Early and transient gene expression changes in pressure overload-induced cardiac hypertrophy in mice. Genomics. 2006;88(4):480-488.

42. Nakamura A, et al. LV systolic performance improves with development of hypertrophy after transverse aortic constriction in mice. Am J Physiol Heart Circ Physiol. 2001;281(3):H1104-H1112.

43. Dobaczewski M, Chen W, Frangogiannis NG. Transforming growth factor (TGF)- $\beta$ signaling in cardiac remodeling. $J$ Mol Cell Cardiol. 2011;51(4):600-606.

44. Andrae J, Gallini R, Betsholtz C. Role of platelet-derived growth factors in physiology and medicine. Genes Dev. 2008;22(10):1276-1312.

45. Kong P, Christia P, Frangogiannis NG. The pathogenesis of cardiac fibrosis. Cell Mol Life Sci. 2014;71(4):549-574

46. Wang X, Ge J, Tredget EE, Wu Y. The mouse excisional wound splinting model, including applications for stem cell transplantation. Nat Protoc. 2013;8(2):302-309.

47. Thulabandu V, Chen D, Atit RP. Dermal fibroblast in cutaneous development and healing. Wiley Interdiscip Rev Dev Biol. 2018;7(2):10.1002/wdev.307. doi: 10.1002/wdev.307.

48. Snider P, Standley KN, Wang J, Azhar M, Doetschman T, Conway SJ. Origin of cardiac fibroblasts and the role of periostin. Circ Res. 2009;105(10):934-947.

49. Coller HA. Cell biology. The essence of quiescence. Science. 2011;334(6059):1074-1075.

50. Tomasek JJ, Gabbiani G, Hinz B, Chaponnier C, Brown RA. Myofibroblasts and mechano-regulation of connective tissue remodelling. Nat Rev Mol Cell Biol. 2002;3(5):349-363.

51. Hortells L, Johansen AKZ, Yutzey KE. Cardiac fibroblasts and the extracellular matrix in regenerative and nonregenerative hearts. J Cardiovasc Dev Dis. 2019;6(3):E29

52. Kanisicak O, et al. Genetic lineage tracing defines myofibroblast origin and function in the injured heart. Nat Commun 2016;7:12260

53. Moore-Morris T, et al. Resident fibroblast lineages mediate pressure overload-induced cardiac fibrosis. J Clin Invest. 2014;124(7):2921-2934.

54. Schiattarella GG, Hill JA. Inhibition of hypertrophy is a good therapeutic strategy in ventricular pressure overload. Circulation. 2015;131(16):1435-1447.

55. Herum KM, Choppe J, Kumar A, Engler AJ, McCulloch AD. Mechanical regulation of cardiac fibroblast profibrotic phenotypes. Mol Biol Cell. 2017;28(14):1871-1882.

56. Solon J, Levental I, Sengupta K, Georges PC, Janmey PA. Fibroblast adaptation and stiffness matching to soft elastic substrates. Biophys J. 2007;93(12):4453-4461.

57. Handorf AM, Zhou Y, Halanski MA, Li WJ. Tissue stiffness dictates development, homeostasis, and disease progression. Organogenesis. 2015;11(1):1-15.

58. Panciera T, Azzolin L, Cordenonsi M, Piccolo S. Mechanobiology of YAP and TAZ in physiology and disease. Nat Rev Mol Cell Biol. 2017;18(12):758-770.

59. Liu F, et al. Mechanosignaling through YAP and TAZ drives fibroblast activation and fibrosis. Am J Physiol Lung Cell Mol Physiol. 2015;308(4):L344-L357.

60. Dupont S, et al. Role of YAP/TAZ in mechanotransduction. Nature. 2011;474(7350):179-183.

61. Calvo F, et al. Mechanotransduction and YAP-dependent matrix remodelling is required for the generation and maintenance of cancer-associated fibroblasts. Nat Cell Biol. 2013;15(6):637-646.

62. Wang P, et al. The alteration of Hippo/YAP signaling in the development of hypertrophic cardiomyopathy. Basic Res Cardiol. 2014;109(5):435.

63. Totaro A, Panciera T, Piccolo S. YAP/TAZ upstream signals and downstream responses. Nat Cell Biol. 2018;20(8):888-899.

64. Brose K, et al. Slit proteins bind Robo receptors and have an evolutionarily conserved role in repulsive axon guidance. Cell. 1999;96(6):795-806.

65. Hohenester E. Structural insight into Slit-Robo signalling. Biochem Soc Trans. 2008;36(Pt 2):251-256.

66. Denk AE, et al. Slit3 inhibits Robo3-induced invasion of synovial fibroblasts in rheumatoid arthritis. Arthritis Res Ther. 2010;12(2):R45

67. Kim H, et al. SLIT3 regulates endochondral ossification by $\beta$-catenin suppression in chondrocytes. Biochem Biophys Res Commun 2018;506(4):847-853.

68. Koh JM. Osteoclast-derived SLIT3 is a coupling factor linking bone resorption to bone formation. BMB Rep. 2018;51(6):263-264

69. Piper M, Georgas K, Yamada T, Little M. Expression of the vertebrate Slit gene family and their putative receptors, the Robo genes, in the developing murine kidney. Mech Dev. 2000;94(1-2):213-217.

70. Li N, et al. Osteoclasts are not a source of SLIT3. Bone Res. 2020;8:11

71. Ducy P, Schinke T, Karsenty G. The osteoblast: a sophisticated fibroblast under central surveillance. Science. 2000;289(5484):1501-1504.

72. Grossman W, Jones D, McLaurin LP. Wall stress and patterns of hypertrophy in the human left ventricle. J Clin Invest. 1975;56(1):56-64.

73. Frey N, Olson EN. Cardiac hypertrophy: the good, the bad, and the ugly. Annu Rev Physiol. 2003;65:45-79.

74. Carabello BA. Is cardiac hypertrophy good or bad? The answer, of course, is yes. JACC Cardiovasc Imaging. 2014;7(11):1081-1083

75. Mekkaoui C, et al. Differential response of the left and right ventricles to pressure overload revealed with diffusion tensor MRI tractography of the heart in vivo. J Cardiovasc Magn Reson. 2015;17(suppl 1):03.

76. Rain S, et al. Right ventricular diastolic impairment in patients with pulmonary arterial hypertension. Circulation. 2013;128(18):2016-2025.

77. Trip P, et al. Clinical relevance of right ventricular diastolic stiffness in pulmonary hypertension. Eur Respir J. 2015;45(6):1603-1612. 78. Bogaard HJ, Voelkel NF. Is myocardial fibrosis impairing right heart function? Am J Respir Crit Care Med. 2019;199(12):1458-1459.

79. Burlew BS, Weber KT. Cardiac fibrosis as a cause of diastolic dysfunction. Herz. 2002;27(2):92-98. 
80. Klein G, et al. Increased collagen deposition and diastolic dysfunction but preserved myocardial hypertrophy after pressure overload in mice lacking PKCepsilon. Circ Res. 2005;96(7):748-755.

81. Gomez-Arroyo J, et al. Iloprost reverses established fibrosis in experimental right ventricular failure. Eur Respir J. 2015;45(2):449-462.

82. Burchfield JS, Xie M, Hill JA. Pathological ventricular remodeling: mechanisms: part 1 of 2. Circulation. 2013;128(4):388-400

83. Gomez-Arroyo J, et al. Differences in right ventricular remodeling secondary to pressure overload in patients with pulmonary hypertension. Am J Respir Crit Care Med. 2014;189(5):603-606.

84. Frey N, Katus HA, Olson EN, Hill JA. Hypertrophy of the heart: a new therapeutic target? Circulation. 2004;109(13):1580-1589.

85. Hill JA, et al. Cardiac hypertrophy is not a required compensatory response to short-term pressure overload. Circulation. 2000;101(24):2863-2869.

86. Esposito G, et al. Genetic alterations that inhibit in vivo pressure-overload hypertrophy prevent cardiac dysfunction despite increased wall stress. Circulation. 2002;105(1):85-92.

87. Kramann R, et al. Perivascular Gli1+ progenitors are key contributors to injury-induced organ fibrosis. Cell Stem Cell. 2015;16(1):51-66.

88. Schultz JE, et al. Fibroblast growth factor-2 mediates pressure-induced hypertrophic response. J Clin Invest. 1999;104(6):709-719.

89. Koide M, et al. Premorbid determinants of left ventricular dysfunction in a novel model of gradually induced pressure overload in the adult canine. Circulation. 1997;95(6):1601-1610.

90. Rogers JH, et al. RGS4 causes increased mortality and reduced cardiac hypertrophy in response to pressure overload. J Clin Invest. 1999;104(5):567-576.

91. Piek A, de Boer RA, Silljé HH. The fibrosis-cell death axis in heart failure. Heart Fail Rev. 2016;21(2):199-211.

92. Takeda N, et al. Cardiac fibroblasts are essential for the adaptive response of the murine heart to pressure overload. J Clin Invest. 2010;120(1):254-265.

93. Shimizu T, et al. Fibroblast deletion of ROCK2 attenuates cardiac hypertrophy, fibrosis, and diastolic dysfunction. JCI Insight. 2017;2(13):93187.

94. Oka T, et al. Genetic manipulation of periostin expression reveals a role in cardiac hypertrophy and ventricular remodeling. Circ Res. 2007;101(3):313-321.

95. Akhurst RJ, Hata A. Targeting the TGF $\beta$ signalling pathway in disease. Nat Rev Drug Discov. 2012;11(10):790-811.

96. Hinz B, Phan SH, Thannickal VJ, Galli A, Bochaton-Piallat ML, Gabbiani G. The myofibroblast: one function, multiple origins. Am J Pathol. 2007;170(6):1807-1816.

97. Koitabashi N, et al. Pivotal role of cardiomyocyte TGF- $\beta$ signaling in the murine pathological response to sustained pressure overload. J Clin Invest. 2011;121(6):2301-2312.

98. Liang M, et al. Yap/Taz deletion in Gli ${ }^{+}$cell-derived myofibroblasts attenuates fibrosis. J Am Soc Nephrol. 2017;28(11):3278-3290.

99. Rognoni E, Walko G. The roles of YAP/TAZ and the Hippo pathway in healthy and diseased skin. Cells. 2019;8(5):E411.

100. Byun J, et al. Yes-associated protein (YAP) mediates adaptive cardiac hypertrophy in response to pressure overload. $J$ Biol Chem. 2019;294(10):3603-3617.

101. Del Re DP. Beyond the Cardiomyocyte: consideration of HIPPO pathway cell-type specificity. Circ Res. 2018;123(1):30-32.

102. Schafer S, et al. IL-11 is a crucial determinant of cardiovascular fibrosis. Nature. 2017;552(7683):110-115.

103. Shah M, Phillips MR, Quintana M, Stupp G, McLean SE. Echocardiography allows for analysis of pulmonary arterial flow in mice with congenital diaphragmatic hernia. J Surg Res. 2018;221:35-42.

104. Tarnavski O, McMullen JR, Schinke M, Nie Q, Kong S, Izumo S. Mouse cardiac surgery: comprehensive techniques for the generation of mouse models of human diseases and their application for genomic studies. Physiol Genomics. 2004;16(3):349-360

105. Zolov SN, et al. In vivo, Pikfyve generates PI(3,5)P2, which serves as both a signaling lipid and the major precursor for PI5P. Proc Natl Acad Sci U S A. 2012;109(43):17472-17477.

106. Ackers-Johnson M, Li PY, Holmes AP, O’Brien SM, Pavlovic D, Foo RS. A simplified, Langendorff-free method for concomitant isolation of viable cardiac myocytes and nonmyocytes from the adult mouse heart. Circ Res. 2016;119(8):909-920.

107. Seluanov A, Vaidya A, Gorbunova V. Establishing primary adult fibroblast cultures from rodents. J Vis Exp. 2010;(44):2033.

108. Adhikari N, et al. Guidelines for the isolation and characterization of murine vascular smooth muscle cells. A report from the International Society of Cardiovascular Translational Research. J Cardiovasc Transl Res. 2015;8(3):158-163. 